\title{
Nitrogen interstitial defects in Silicon. A quantum mechanical investigation of the structural, electronic and vibrational properties.
}

\author{
Alexander Platonenko, ${ }^{1}$ Francesco Gentile, ${ }^{2}$ Jefferson Maul, ${ }^{2}$ \\ Fabien Pascale, ${ }^{3}$ Eugene A Kotomin, ${ }^{1}$ and Roberto Dovesi ${ }^{2}$ \\ ${ }^{1}$ Institute of Solid State Physics, University of Latvia, \\ 8 Kengaraga street, LV1063, Riga, Latvia \\ ${ }^{2}$ Dipartimento di Chimica, Università di Torino and \\ NIS (Nanostructured Interfaces and Surfaces) Centre, \\ Via P. Giuria 5, 10125 Torino, Italy \\ ${ }^{3}$ Université de Lorraine - Nancy, CNRS, \\ Laboratoire de Physique et Chimie Théoriques, \\ UMR 7019. Vandouvre-lès-Nancy, 54506 France
}




\begin{abstract}
The vibrational features of eight interstitial nitrogen related defects in silicon have been investigated at the first principles quantum mechanical level by using a periodic supercell approach, a hybrid functionals, an all electron Gaussian type basis set and the CRYSTAL code. The list includes defects that will be indicated as $\mathrm{N}_{i}$ (one $\mathrm{N}$ atom forming a bridge between two Si atoms), $\mathrm{N}_{i}-\mathrm{N}_{s}$ (one interstitial and one substitutional $\mathrm{N}$ atom linked to the same $\mathrm{Si}$ atom), $\mathrm{N}_{i}-\mathrm{N}_{i}$ (two $\mathrm{N}_{i}$ defects linked to the same couple of silicon atoms) and $\mathrm{N}_{i}-\mathrm{Si}_{i}-\mathrm{N}_{i}$ (two $\mathrm{N}_{i}$ defects linked to the same interstitial silicon atom). Four $\langle 001\rangle$ split interstitial (dumbell) defects have also been considered, in which one lattice atom splits in two, and as a result the two interstitial atoms are three fold coordinated: they are two $\mathrm{N}$ (indicated as $\mathrm{I}_{\mathrm{N}-\mathrm{N}}$ ), one $\mathrm{N}$ and one $\mathrm{Si}\left(\mathrm{I}_{\mathrm{N}-\mathrm{Si}}\right)$, one $\mathrm{N}$ and one $\mathrm{C}\left(\mathrm{I}_{\mathrm{C}-\mathrm{N}}\right)$. For comparison, also the case with two $\mathrm{Si}$ atoms $\left(\mathrm{I}_{\mathrm{Si}-\mathrm{Si}}\right)$ has been included. Four of these eight defects have unpaired electrons, and have been described through the UHF (Unrestricted Hartree-Fock like) computational scheme. The local defect geometry and the charge and spin density distributions have been analyzed. For the first time, intensities of IR and Raman spectra were calculated along with the frequencies, and this is crucial for the comparison of theoretical simulations with experiments. All these defects present very characteristic features in their IR spectrum, dominated by one or two very intense peaks. It has been possible to find a simulated counterpart to each one of the five peaks reported by Stein in 1985 (Applied Physics Letters, 47, 1339), and then to establish a correspondence between the microscopic structure of the defects and the IR intense peaks. The Raman spectra are in all cases dominated by the perfect silicon peak at about $530 \mathrm{~cm}^{-1}$, and are then not very useful for the characterization of the defects.
\end{abstract}

Keywords: Silicon, Point defects, Nitrogen defects, Infrared Spectra, Raman Spectra, CRYSTAL code 


\section{INTRODUCTION}

Electronic and mechanical properties of pristine silicon are known to be significantly affected by the presence of impurities and defects. Nitrogen doping improves silicon properties in various ways, increasing mechanical strength and greatly reducing the size and density of void defects ${ }^{1-3}$. It has been observed that nitrogen increases precipitation of oxygen by increasing the number of nucleation sites and retarding silicon self-interstitial migration ${ }^{4}$. Diffusivity of N-N dimers was found to be three orders of magnitude higher as compared to oxygen at $1100{ }^{\circ} \mathrm{C}$ (see Ref. 5). N-N pairs were reported to form stable complex with Si vacancies, and in this way vacancy aggregation is suppressed ${ }^{6,7}$. When both nitrogen and carbon were implanted in silicon, it was observed that diffusion of both species was affected. This could be the result of a nitrogen-nitrogen pair breakdown and formation of C-N defect complexes ${ }^{8}$.

Nitrogen can be introduced into $\mathrm{Si}$ by adding $\mathrm{Si}_{3} \mathrm{~N}_{4}$ to the $\mathrm{Si}$ melt and annealing in an atmosphere of $\mathrm{N}_{2}$ or $\mathrm{NF}_{3}{ }^{9,10}$. Nitrogen solubility in silicon remains however very small, so concentration of natural $\mathrm{N}$ defects is low in standard conditions, making experimental measurements difficult. High N content can be obtained through implantation techniques and subsequent pulsed laser annealing ${ }^{11}$. Thus increased concentration of $\mathrm{N}$ atoms permits accurate infrared (IR) measurements. Stein's IR spectra ${ }^{12,13}$, obtained along these lines, present characteristic peaks at 551,653, 687, 766 and $963 \mathrm{~cm}^{-1}$.

Quantum mechanical computer simulations represent the natural alternative to experiment, in particular when the latter is difficult, as in the present case due to low $\mathrm{N}$ concentration, or for other reasons. In 1994 Jones et al. ${ }^{14}$ computed the vibrational frequencies of various defects by using the quantum cluster approach $\left(\mathrm{N}_{2} \mathrm{Si}_{44} \mathrm{H}_{42}\right)$ and a LDA type functional. By combining quantum mechanical calculations, IR spectroscopy and channeling, an interpretation of the dominant Stein peaks ${ }^{12,13}$ was proposed. Nine years later an extensive simulation of the vibrational properties of many defects in silicon was performed by Goss et al. ${ }^{15}$, by using a quantum-mechanical approach, Gaussian type functions and pseudopotentials, a GGA type functional and a supercell scheme. The two Stein's peaks at high wavenumber $\left(766\right.$ and $963 \mathrm{~cm}^{-1}$ ) were attributed by Goss to a couple of interstitial (as opposed to substitutional) N atoms, belonging to a square configuration. Note that intrinsic interstitial defects are very common in all kinds of materials (metals, semiconductors, insulators) and 
often are produced as a result of irradiation ${ }^{16}$. These defects control many material properties including electrical conductivity and radiation stability.

In a complementary paper by some of the present authors ${ }^{17}$, seven nitrogen substitutional defects in silicon have been investigated with exactly the same computational ingredients adopted for the present study, namely a hybrid functional (B3LYP), a local Gaussian type basis set, the supercell scheme and the CRYSTAL code. The aim of both papers is to explore the possibility of a clear, individual characterization of these defects on the basis of the vibrational IR and Raman spectra. For the first time the IR and Raman intensities, that are computed analytically, were obtained for this kind of defects. The main results of Reference 17 can be summarized as follows:

a) in all cases the IR spectrum is dominated by a single (or a couple of) peak, well separated from the silicon bulk vibrational band.

b) all the high intensity peaks of these substitutional defects fall in the $645-712 \mathrm{~cm}^{-1}$ range.

c) as a consequence, the peaks observed by Stein ${ }^{12,13}$ at $765 \mathrm{~cm}^{-1}$ and $962 \mathrm{~cm}^{-1}$ cannot be attributed to one of the various substitutional nitrogen defects.

d) Only two of the three remaining Stein's peaks (the ones at 653 and $687 \mathrm{~cm}^{-1}$ ) can be attributed to substitutional and/or substitutional plus vacancy defects.

d) The peak at $551 \mathrm{~cm}^{-1}$ does not find then any theoretical counterpart ${ }^{17}$ : none of the substitutional investigated defects presents an intense peak in that region.

In this work, we extend the previous analysis to eight defects containing nitrogen in interstitial positions, trying also to complete the identification of Stein's peaks. A first set of defects (see Figure 3) includes $\mathrm{N}_{i}$ (an $\mathrm{N}$ atom connecting two Si atoms), and three double or triple defects related to it: $\mathrm{N}_{i}-\mathrm{N}_{s}$ (one $\mathrm{N}_{i}$ and one substitutional $\mathrm{N}_{s}$ linked to the same Si atom), $\mathrm{N}_{i}-\mathrm{N}_{i}$ (two $\mathrm{N}_{i}$ linked to the same couple of $\mathrm{Si}$ atom), and $\mathrm{N}_{i}-\mathrm{Si}_{i}-\mathrm{N}_{i}$ (two $\mathrm{N}_{i}$ atoms linked to the same interstitial Si atom). A second set (see Figure 1) includes four $\langle 100\rangle$ split intestitial defects, in which two atoms are threefold coordinated. They can be both $\mathrm{N}$ atoms $\left(\mathrm{I}_{\mathrm{N}-\mathrm{N}}\right)$, one $\mathrm{N}$ and one $\mathrm{Si}\left(\mathrm{I}_{\mathrm{N}-\mathrm{Si}}\right)$, one $\mathrm{N}$ and one $\mathrm{C}\left(\mathrm{I}_{\mathrm{C}-\mathrm{N}}\right)$ or two Si atoms $\left(\mathrm{I}_{\mathrm{Si}-\mathrm{Si}}\right.$ : this defect has been added for completeness). The IR and Raman intensities are evaluated analytically, and the full spectra can be generated and compared with experiment, when available. It should be underlined that previous simulations ${ }^{14,15}$ were limited to the vibrational frequencies, without the IR and Raman intensities, therefore, no indication at all is provided concerning the number of important peaks that should appear in the spectrum, 
their intensity (null, small, medium, large), and then their correlation with the presence of various possible defects.

The paper is structured as follows. Section II contains the details of the computational procedures and conditions. Section III is organized in three parts: Subsection III A describes the fully-relaxed structures of the defective lattices, their charge (and spin) distributions, the band structure and the formation energy. Subsection III B reports the corresponding vibrational features and their detailed analysis. In Section IV the present results are discussed, and compared with experiments and previous calculations. Finally Section V contains the conclusions of this study.

\section{COMPUTATIONAL DETAILS}

Calculations have been performed by use of the B3LYP global hybrid functional, ${ }^{18,19}$ as implemented in the CRYSTAL17 program. ${ }^{20}$ An all-electron Gaussian type basis set (derived from Pople's standard 6-31G ${ }^{21}$ ) as described in D'Arco etal. ${ }^{22}$ has been adopted for silicon: the contraction is $6-5111 \mathrm{G}^{*}$ (22 atomic orbitals, AOs), where the first shell is of $s$ type, followed by $4 s p$ and one d shell; the exponents of the two outermost $s p$ shells have been set to $0.45 \mathrm{Bohr}^{-2}$ and $0.15 \mathrm{Bohr}^{-2}$, respectively. For the nitrogen and carbon atoms, the 6- $31 \mathrm{G}^{* 23}$ contraction has been adopted without modifications (14 AOs both).

The truncation of the Coulomb and exchange infinite lattice series is controlled by five thresholds $\mathrm{T}_{i}$ (see CRYSTAL manual, Ref. 24, for more details), which have been set to $8\left(\mathrm{~T}_{1}-\mathrm{T}_{4}\right)$ and $16\left(\mathrm{~T}_{5}\right)$. The convergence threshold on energy for the self-consistent-field (SCF) procedure has been set to $10^{-8}$ and $10^{-11}$ Hartree for the structural and frequency calculations, respectively.

The DFT exchange-correlation contribution to the Fock matrix has been evaluated by numerical integration over the unit cell volume. Radial and angular points for the integration grid are generated through Gauss-Legendre radial quadrature and Lebedev two-dimensional angular point distributions. The default pruned grid with 74 radial and 974 angular points has been used. An estimate of the accuracy of this grid is given by the number $\mathrm{n}_{i}$ of electrons obtained by integration of the charge density: in the case of the $\mathrm{I}_{\mathrm{N}-\mathrm{N}}$ defect, $n_{i}=894.003$, to be compared to the total number of electrons in the unit cell (894).

A periodic super-cell approach is used to simulate different defect concentrations. Cubic 
super-cells containing 64 and 216 atoms have been considered (to be referred to in the following as $S_{n}$, with $n=64$ or 216). They correspond to the expansion of the bulk silicon conventional cell, containing 8 atoms, by a factor 2 and 3 along the three lattice parameters. The point defects reduce the symmetry; the deformation of the cell from the cubic shape is however negligible. Overall, the size of the basis set (number of AOs) is 1408 in $S_{64}$ and 4752 in $S_{216}$ (these numbers refer to the perfect supercell). Reciprocal space has been sampled using a regular sub-lattice with a shrinking factor of 4 for $S_{64}$ and of 2 for $S_{216}$; this corresponds to a number of $\mathbf{k}$ points in the irreducible part of Brillouin zone varying from 4 to 36 , according to the point symmetry and supercell size.

The relevant (defect) peak positions vary at most by $1-3 \mathrm{~cm}^{-1}$ in going from $\mathrm{S}_{64}$ to $\mathrm{S}_{216}$. In the following all data refer to the smallest supercell.

\section{A. Harmonic frequencies and the IR and Raman spectra}

Frequencies at the $\Gamma$ point are obtained within the harmonic approximation by diagonalising the mass-weighted Hessian matrix, $W$, whose elements are defined as ${ }^{25-29}$

$$
W_{\alpha i, \beta j}^{\Gamma}=\frac{H_{\alpha i, \beta j}^{\mathbf{0}}}{\sqrt{M_{\alpha} M_{\beta}}} \quad \text { with } \quad H_{\alpha i, \beta j}^{\mathbf{0}}=\left(\frac{\partial^{2} E}{\partial u_{\alpha i}^{\mathbf{0}} \partial u_{\beta j}^{\mathbf{0}}}\right),
$$

where $M_{\alpha}$ and $M_{\beta}$ are the masses of atoms associated with the $i$ and $j$ atomic coordinates. Once the Hessian matrix, $\mathcal{H}_{\alpha i, \beta j}$, has been calculated, frequency shifts due to isotopic substitutions can be calculated readily, at no computational cost, by changing masses in Equation 1.

Energy first derivatives with respect to the atomic positions, $v_{\alpha, j}=\partial E / \partial u_{\alpha, j}$, are calculated analytically for all the $u_{\alpha, j}$ coordinates ( $E$ is the total energy, $u_{\alpha, j}$ is the displacement coordinate with respect to the equilibrium, $\alpha$ labels each atom), whereas second derivatives at $\vec{u}=0$ are calculated numerically using a single displacement along each coordinate $(N=2$, the central point and a point on the right side of the parabola):

$$
\left[\frac{\partial v_{\alpha j}}{\partial u_{\beta i}}\right] \approx \frac{v_{\alpha j}\left(0, \ldots, u_{\beta i}, \ldots\right)}{u_{\beta i}}
$$

or averaging two displacements $(N=3)$ :

$$
\left[\frac{\partial v_{\alpha j}}{\partial u_{\beta i}}\right] \approx \frac{v_{\alpha j}\left(0, \ldots, u_{\beta i}, \ldots\right)-v_{\alpha j}\left(0, \ldots,-u_{\beta i}, \ldots\right)}{2 u_{\beta i}}
$$


Previous calculations ${ }^{25,30}$ have shown that in bulk systems the influence of both $u$ and $N$ is very small (less than $1 \mathrm{~cm}^{-1}$ ) when $\mathrm{H}$ atoms are not present.

Integrated intensities for IR absorption $\mathcal{I}_{p}$ are computed for each mode $p$ by means of the mass-weighted effective-mode Born-charge vector $\vec{Z}_{p}^{31,32}$ evaluated through a CPHF/KS approach: $:^{33,34}$

$$
\mathcal{I}_{p} \propto\left|\vec{Z}_{p}\right|^{2}
$$

The relative Raman intensities of the peaks are computed analytically by exploiting a scheme illustrated in refs. 35,36. Both schemes are based on the solutions of first- and second-order Coupled-Perturbed-Hartree-Fock/Kohn-Sham (CPHF/KS) equations. ${ }^{37,38}$

\section{B. Optimization strategy and symmetry for the $\langle 001\rangle$ split interstitial defects}

The conventional unit cell of bulk silicon, in which the ideal structure of the $\langle 001\rangle$ split interstitial defect is inserted, is shown in Figure 1. This defect structure can be seen as due to the insertion, along the $\langle 001\rangle$ direction, of an additional atom (B) that pushes one lattice atom (A) upwards.

If $\mathrm{A}$ and $\mathrm{B}$ are chemically equivalent (two $\mathrm{N}$ or two Si atoms in our case) the symmetry can be $\mathrm{D}_{2 d}$, see Figure 2, left. The principal axis is $\mathrm{S}_{4}$, with two $\mathrm{C}_{2}$ axes orthogonal to it, and two $\sigma$ planes. Overall 8 symmetry operators, five classes and five Irreducible Representations. If the two atoms are different, the symmetry reduces to $\mathrm{C}_{2 v}$. However, the size of the interstitial atoms can be such that one of them, or both, prefers to move from the $\mathrm{S}_{4}$ or $\mathrm{C}_{2}$ axis, with a consequent reduction of symmetry, as documented in the Sym column of Table I. We remind that in the optimization process, the CRYSTAL code (as many other codes) stops when the gradients and the displacements (the largest one and the root-mean-square of the full set) are smaller than a given threshold. However, a stationary point (a point in which the gradient is null) might correspond to a maximum, a minimum or to a saddle point. Typically, a constrained optimization (imposing a given symmetry, for example) can bring to a saddle point. The calculation of the Hessian and its diagonalization in this point will produce then one or more imaginary frequencies.

The strategy that has been followed in the present investigation for avoiding the false minima is then the following:

a) Perform a geometry optimization with the $\mathrm{D}_{2 d}$ or $\mathrm{C}_{2 v}$ symmetry. 
b) Perform the frequency calculation at this constrained minimal energy geometry.

c1) If all frequencies are positive, this is the real minimum.

c2) If there is one or more imaginary frequency, follow (by using the SCANMODE option ${ }^{24}$ ) the corresponding eigenvector (or one of the eigenvectors, if more than one imaginary frequency appears); Figure 4 provides some examples. The symmetry of the mode automatically defines the subgroups of the original group. This subgroup is determined automatically by the code.

d) As point a) was a maximum with respect to the normal coordinate with imaginary frequency, a minimum along this coordinate is determined with SCANMODE.

e) Starting from the minimum determined in d), a new full optimization is performed in the full coordinate space and reduced point symmetry.

f) A new frequency calculation is performed, that in all cases leads to the full set of positive frequencies.

In all the cases here considered, no more than a single imaginary frequency was found when imposing the high symmetry.

It should be noticed that in many similar investigations published on the literature, only step a) (or possibly, and rarely, steps a) and b)) is performed, so that the evidence that the stationary point is a real minimum is not provided, and this can generate severe errors in the subsequent analysis.

\section{RESULTS}

\section{A. Geometry, charge and spin densities, and band structure}

1. The $N_{i}, N_{i}-N_{s}, N_{i}-N_{i}$ and $N_{i}-S i_{i}-N_{i}$ defects

The structure of the $\mathrm{N}_{i}$ defect is shown in Figure 3, top. Here the nitrogen atom, at variance with respect to many other $\mathrm{N}$ containing defects, in only twofold coordinated, with three extra electrons, two in the lone pair, and one in the direction perpendicular to the plane defined by Si-N-Si. The N-Si distances $1.66 \AA$, the Si-N-Si angle is $148.6^{\circ}$. The uncoupled electron is mostly localized on $\mathrm{N}(\mu=+0.651|e|)$. The two Si first neighbors have very small and negative spin density $(\mu=-0.03|e|)$, whereas the six second neighbors carry positive spin density (about $+0.06|e|$ each); this brings the total spin momentum very close to the 
expected $\mu=+1|e|$ value. The net charge $q$ on the $\mathrm{N}$ atom is equal to $-0.763|e|$, that is nearly exactly compensated by the two nearest silicon atoms with net charge of $+0.391|e|$. The N-Si bond populations $(+0.296$ and $+0.272|e|)$ indicate a strong covalent bond. The band structure in shown in Figure 5. The defect energy level is just above the valence band, whereas the empty antibonding level is in the middle of the band gap (0.95 eV above the top of the valence band at the $\Gamma$ point). Figure 6 shows that the spin density is very localized around $\mathrm{N}$, as expected, on a couple of p orbitals.

It is interesting to compare the electronic structure of the $\mathrm{N}_{i}$ - $\mathrm{N}_{s}$ defect (see second row in in Figure 3) with the one of the two isolated $\left(\mathrm{N}_{i}\right.$ and $\left.\mathrm{N}_{s}\right)$ defects. Both $\mathrm{N}_{i}$ and $\mathrm{N}_{s}$ are characterized by an uncoupled electron. When close to each other, however, they are able to couple the two electrons, giving rise to a closed shell solution. The two N-Si distances are 1.62 and $1.67 \AA$, to be compared to 1.66 and $1.68 \AA$ in $\mathrm{N}_{i}$; the bond populations are +0.403 and $+0.332|e|$, to be compared to 0.272 and $0.296|e|$; both bonds are stronger than in the isolated $\mathrm{N}_{i}$ defect; the shorter one is closer to a double bond than to a single bond. The bond population between $\mathrm{N}_{s}$ and its neighbors is around $+0.14|e|$, indicating that these bonds are semi-ionic (or weak covalent bonds). The net charge of the two $\mathrm{N}$ atoms is very close to $-1|e|$; the $q$ charge on the common silicon atom is $+0.725|e|$, whereas it is +0.430 $|e|$ on $\mathrm{Si}$ linked to $\mathrm{N}_{i}$, and $+0.350|e|$ on the three $\mathrm{Si}$ atoms linked to $\mathrm{N}_{s}$. Overall, the defect region including the two $\mathrm{N}$ atoms and their $5 \mathrm{Si}$ neighbors is nearly neutral. The band structure is shown in Figure 5. The doubly occupied defect levels appear just above the top of the valence band, whereas the two empty antibonding levels are about $1.4 \mathrm{eV}$ above, in the middle of the band gap.

The last two studied defects contain a couple of nitrogen interstitials. The latter defect includes also a silicon interstitial atom. The structures are shown in Figure 3, third and bottom rows. In $\mathrm{N}_{i}-\mathrm{N}_{i}$, two nitrogen and two silicon atoms form an almost ideal square structure. The two $\mathrm{N}$ atoms are threefold coordinated, and then the solution turns out to be a closed shell. The Si-N bonds are 1.74 and $1.78 \AA$ long, longer than the one in the isolated $\mathrm{N}_{i}(1.66 \AA)$, but shorter than in $\mathrm{N}_{s}(1.86 \AA)$. This defect is known to be one of the most stable N-related defects in silicon ${ }^{14}$. Also the bond populations are intermediate between the ones of isolated $\mathrm{N}_{i}$ and $\mathrm{N}_{s}:+0.24|e|$ in the square $\left(+0.14|e|\right.$ in $\mathrm{N}_{s}$ and +0.29 $|e|$ in $\mathrm{N}_{i}$ ). The square defect is quite ionic: the net charge is $-1.106|e|$ on $\mathrm{N}$, and $+0.851|e|$ on Si. The charge drops down to $+0.432|e|$ on the external Si atoms linked to N. The high 
stability of this structure is confirmed by the band structure, characterized by a band gap as large as $2.27 \mathrm{eV}$, the largest among the ones shown in Figure 5, referring to all defects here considered. The defect levels appear just above the top of the valence band.

When an additional silicon interstitial atom is inserted in the structure (bottom row of Figure 3), the geometry is largely altered. The two nitrogen atoms are threefold coordinated and silicon is fourfold coordinated, so the system is closed shell. The three interstitial atoms push away the surrounding atoms from their initial positions. The band gap is as large as $2.18 \mathrm{eV}$ (see the bottom right panel of Figure 5); the defective level appears just above the top of the valence band. The Si-N bonds are from 1.73 to $1.79 \AA$ long. The Si-N bonds are strongly polarized: the net charge is about $-1.107|e|$ on $\mathrm{N}$, and $+0.801|e|$ on Si. The defect cluster containing the interstitial atoms and their first neighbors is however nearly neutral $(+0.20|e|)$.

\section{Nitrogen split-interstitial defects $I_{N-N}, I_{N-S i}$ and $I_{C-N}$}

The geometry and charge and spin density data, as resulting from a Mulliken analysis, of the $\mathrm{I}_{\mathrm{X}-\mathrm{Y}}$ defects are summarized in Table I.

The point symmetry of $\mathrm{I}_{\mathrm{N}-\mathrm{N}}$ is $\mathrm{D}_{2 d}$. The ground state is a closed shell function, as the nitrogen atom can distribute his five electrons in the three covalent bonds and in a lone pair, that in the present case is orthogonal to the plane defined by A, E and E' (or B, $\mathrm{D}$ and D', see figure 1). Note that these two planes are orthogonal to each other (hence the $\mathrm{S}_{4}$ symmetry). The $\mathrm{N}$ net charge is $-0.721|e|$, and the one of the $\mathrm{E}$ (and $\mathrm{D}$ ) atoms is $+0.427|e|$; so the total charge of the $\mathrm{N}_{2} \mathrm{Si}_{4}$ cluster is $+0.266|e|$; the 12 second neighbors are slightly negatively charged, so that the overall charge of the $\mathrm{N}_{2} \mathrm{Si}_{14}$ cluster reduces to $+0.084|e|$. The N-N distance is $1.46 \AA$ and the A-B bond population is equal to $+0.12|e|$ (weak covalent bond). The equilibrium geometry and the electronic structure are strongly conditioned by the geometrical constraints, and are then far away from the single bond N-N situation in a molecular context. For comparison, let us consider the hydrazine molecule, $\mathrm{N}_{2} \mathrm{H}_{4}$, treated at the same level of theory, and using for $\mathrm{N}$ the same basis set here adopted (for H a 31G Pople's set has been used). The optimized N-N distance is longer (1.49 vs 1.46 $\AA)$ and the bond populations larger $(+0.20$ versus $+0.12|e|)$ than in $\mathrm{I}_{\mathrm{N}-\mathrm{N}}$. More important, the $\mathrm{N}$ hybridization in the molecule is $s p^{3}$, whereas in the defect is $s p^{2}$. When forcing the 
molecule to assume an $\mathrm{S}_{4}$ geometry, as in the bulk defect, the energy increases by $1.2 \mathrm{eV}$ and the N-N distance reduces to $1.36 \AA$. In summary, the spatial freedom of the molecule generates a local structure of the $\mathrm{N}-\mathrm{N}$ couple that is completely different from the defective one. The band structure of $\mathrm{I}_{\mathrm{N}-\mathrm{N}}$ is shown in figure 5 (first row, central panel). The lone pair levels of the nitrogen atoms fall down in the valence band; the corresponding virtual levels appear just at the lower border of the conduction band.

Let us consider now the $\mathrm{I}_{\mathrm{N}-\mathrm{Si}}$ defect. The Mulliken net charge on $\mathrm{N}$ is -1.05 $|e|$ (it was $-0.721|e|$ in $\mathrm{I}_{\mathrm{N}-\mathrm{N}}$ ); the $\mathrm{Si}$ (atom B in Figure 1) net charge is $+0.350|e|$; the net charges of the D, D', E, E' silicon atoms are $+0.001,+0.001,+0.442,+0.416$, so that overall the $\mathrm{NSi}_{5}$ defect cluster charge is $+0.16|e|$. The Si second neighbors are negatively charged, so that the cluster containing the 18 atoms closest to the defect is essentially neutral (the residual charge is $+0.04|e|)$. The A-B distance is $1.73 \AA$ (see table I; the A-E (N-Si) and B-D (Si-Si) are 1.78 and $2.29 \AA$, respectively ( remember that the silicon atom has a much larger atomic radius than $\mathrm{N}$ ). The $\mathrm{A}-\mathrm{B}$ bond population is $+0.17|e|$; it increases to +0.22 $|e|$ and $0.32|e|$ for A-E and B-D.

The Mulliken magnetic moment $\mu$ is $+0.674|e|$ on the $\mathrm{B}(\mathrm{Si})$ atom, and only $+0.004|e|$ on $\mathrm{N}$. The $\mu$ values on D,D', E and E' are $-0.011,-0.011,+0.010,-0.004$, so that overall the $\mathrm{NSi}_{5}$ cluster contains $0.662|e|$ of the uncoupled electron. Many atoms at larger distance from the defect do have fractions of the spin density necessary to reach one electron. The band structure of $\mathrm{I}_{\mathrm{N}-\mathrm{Si}}$ is shown in figure 5 (first row, right panel). The occupied defect level is $0.44 \mathrm{eV}$ above the top of the valence band (at the $\Gamma$ point); the empty defect level is 0.43 below the bottom of the conduction band (again this value refers to $\Gamma$ ).

In the $\mathrm{I}_{\mathrm{C}-\mathrm{N}}$ defect, the $\mathrm{C}-\mathrm{N}$ distance is $1.45 \AA$ and the corresponding bond population $\mathrm{b}$ is $+0.19|e|$. The C-Si bond population is $+0.36|e|$ (very strong covalent bond), and $\mathrm{b}_{C-N}$ $=+0.21|e|$. The carbon net charged is very small $(-0.082|e|)$ and negative; it is as large as $-0.937|e|$ on nitrogen. The magnetic moment $\mu$ is localized on the $\mathrm{C}$ atom, as expected $(+0.83|e|)$.

The band structure (Figure 5) is shown in the first row, left panel. In this case there is an occupied defect level just above the top of the valence band $(0.1 \mathrm{eV}$ at $\Gamma)$, and a corresponding virtual level at about $0.4 \mathrm{eV}$ below the conduction band. The spin density map (top row, right panel of figure 6) shows that the spin density around the $\mathrm{C}$ atom is more compact than for the Si atom, as a consequence of the size of the p orbitals in the 
two cases.

3. Silicon split-interstitials $I_{S i-S i}$ in triplet and singlet states

The above discussion clearly suggests that when two Si atoms are involved in the interstitial defect, the $\mathrm{D}_{2 d}$ (for the triplet) and $\mathrm{C}_{2 v}$ (for the singlet) symmetry is lost, as the two $\mathrm{Si}$ atoms move from the $\mathrm{C}_{2}$ axis. The energy difference between the optimized geometry when the high symmetry is conserved or released is as large as $0.648 \mathrm{eV}$ for the singlet, and $0.159 \mathrm{eV}$ for the triplet (see Table III). The final energy difference is given in table I. The singlet is more stable by $5 \mathrm{meV}$ as a consequence of the short range Pauli-type repulsion. The band structure of $\mathrm{I}_{\mathrm{Si}-\mathrm{Si}}$ in the singlet $s$ and triplet $t$ states are shown in the bottom row of Figure 5 .

In the $t$ case there are two occupied defect bands in the gap $(0.13 \mathrm{eV}$ above the top of the valence band at $\Gamma$ ), and two empty defect bands separated by $0.41 \mathrm{eV}$ from the bottom of the conduction band. The corresponding numbers for the $s$ state are 0.24 and $0.43 \mathrm{eV}$. The two occupied bands are separated by 0.17 and $0.08 \mathrm{eV}$ from the virtual bands in $t$ and $s$, respectively. In $t$ the two occupied bands are separated by $0.17 \mathrm{eV}$, whereas in $s$ they are nearly degenerate. The spin density map, shown in the left bottom panel of Figure 6 for the $t$ state, shows that: i) the electron is strongly localized in a $p$ type orbital; ii) the unpaired electron on the second defect Si atom is located in a $p$ orbital orthogonal to the plane of the figure; iii) trace of the orthogonality of the orbitals containing the two unpaired electrons is visible in the zero and negative isodensity line along the Si-Si connecting line.

\section{Formation energy}

Defect formation energies $E_{f}$ are reported in Table $\mathrm{V}$, and were calculated according to the following equation:

$$
E_{f}=\left(E_{D}-n * E_{N}\right)-\left(E_{P}-m * E_{S i}\right)
$$

where $E_{D}$ and $E_{P}$ are the total energies of the defective and pristine 64-atoms silicon supercell, $n$ and $m$ are the numbers of atoms added or removed to the defective supercell, 
$E_{N}$ is half the energy of two nitrogen atoms in their most stable form, i.e. the $\mathrm{N}_{2}$ molecule $(-54.73426 \mathrm{Ha}), E_{S i}$ is the total energy of the silicon atom in the pristine silicon structure (that is, half the electronic total energy of the primitive cell, that with the present basis set and functional is $-289.46807 \mathrm{Ha})$.

$\mathrm{E}_{f}^{1}$ is the formation energy per unit cell, whereas $\mathrm{E}_{f}^{1} / \mathrm{X}$ is the same quantity per nitrogen atom. As the $\mathrm{N}_{i}-\mathrm{N}_{i}$ defect turns out to be the most stable, in the $\delta E$ column the $\mathrm{E}_{f}^{1} / \mathrm{X}$ data are shifted so that the $\mathrm{N}_{i}-\mathrm{N}_{i}$ defect appears at zero energy. A few more comments:

a) the process of formation of all defects is endothermic, if reference is done to the $\mathrm{N}_{2}$ molecule

b) The $\mathrm{E}_{f}^{2}$ and $\mathrm{E}_{f}^{2} / \mathrm{X}$ columns report the same data as $\mathrm{E}_{f}^{1}$ and $\mathrm{E}_{f}^{1} / \mathrm{X}$, but shifted by 4.72 $\mathrm{eV}$, half the formation energy of $\mathrm{N}_{2}$ times the number of nitrogen atoms in the unit cell. This means that in equation 5 reference is set to the isolated nitrogen atom. In this case all processes are exothermic.

c) $\mathrm{I}_{N-N}$ is the second more stable (1.16 eV above $\mathrm{N}_{i}-\mathrm{N}_{i}$ ), followed by $\mathrm{N}_{i}-\mathrm{N}_{s}$ and $\mathrm{N}_{i}-\mathrm{Si}_{i}-\mathrm{N}_{i}$ (1.64 and $1.66 \mathrm{eV}$, respectively).

d) $\mathrm{I}_{S i-N}$ and $\mathrm{N}_{i}$ are even less stable $(2.27$ and $2.89 \mathrm{eV})$

e) in spite of the many differences between the present computational method and the one adopted by Goss et al., the two sets of formation energies are similar, and in particular the relative order of stability is about the same. The largest difference between the two $\delta E$ columns is $0.66 \mathrm{eV}$, and refers to $\mathrm{N}_{i}$.

\section{B. Spectroscopic characterization}

The experimental Raman spectrum of pristine silicon ${ }^{39}$ is characterized by a single, three fold degenerate peak at $521 \mathrm{~cm}^{-1}$. The calculated peak with the present functional and basis set is at $533 \mathrm{~cm}^{-1}, 12 \mathrm{~cm}^{-1}$ above, see the bottom panel of figure 7 . The IR spectrum is even simpler: it is completely flat. So any observed IR signal or any Raman peak other than the one at $521 \mathrm{~cm}^{-1}$ must be attributed to defects.

As the mass of nitrogen is nearly exactly half the one of silicon (14.01 vs $28.09 \mathrm{u}$ ), we can anticipate that many of the modes to which $\mathrm{N}$ contributes will appear well above the phonon band of pristine silicon, that has a calculated higher limit at $533 \mathrm{~cm}^{-1}$ (see top panel of Figure 7). Besides that, the N-Si bonds are extremely polar (in most of the cases 
the net charge of $\mathrm{N}$ is close to $-1|e|)$; as a consequence, the IR defect peaks are expected to be extremely intense, and to dominate the spectrum.

The identification of the nature of the modes (which atom is contributing? what kind of movement is described by the mode?) can easily be performed through two tools implemented in the CRYSTAL code:

a) the isotopic shift (see the note following eq. 1); at negligible computational cost the mass of a subset of atoms can be altered arbitrarily. Note that at variance with respect to experiment, i) only very particular atoms of the cell can be involved in the isotopic substitution; ii) the attributed mass is not required to correspond to an existing isotope. Very large masses can be attributed to atoms in order to magnify the mass effect.

b) the graphical animation of the modes. This permits to have a direct and clear evidence of the atoms and groups providing important contributions to the eigenvectors; it is available at the website http://www.crystal.unito.it/animations-of-vibrational-modes.php

\section{The $N_{i}, N_{i}-N_{i}, N_{i}-N_{s}$ and $N_{i}-N_{i}-S i_{i}$ defects}

The IR spectrum of the interstitial nitrogen defect, $\mathrm{N}_{i}$, is characterized by a single dominant peak at $1165 \mathrm{~cm}^{-1}$ (se Figure 8, top). There is a second peak above the top of the vibrational band of pure silicon at $533 \mathrm{~cm}^{-1}$ : it appears at $606 \mathrm{~cm}^{-1}$, with an intensity at least 30 times smaller than the one of the dominant peak.

The mode at $1165 \mathrm{~cm}^{-1}$ is also Raman active. The defect peak height is about half that of the perfect silicon peak, that in this case appears at 524 (instead of 533) $\mathrm{cm}^{-1}$.

The IR spectrum of $\mathrm{N}_{i}-\mathrm{N}_{i}$ presents three intense peaks, separated by about $200 \mathrm{~cm}^{-1}$ : at 563, 782 and $971 \mathrm{~cm}^{-1}$. The first mode is associated with a Si-Si motion and almost not affected by the ${ }^{15} \mathrm{~N}$ isotopic substitution; the two other modes are Si-N stretching and N-N motion perpendicular and parallel to the N-Si-N-Si square diagonal, respectively.

The second row panels of Figure 8 refers to the mixed $\mathrm{N}_{i}-\mathrm{N}_{s}$ defect. Also in this case there are three dominant peaks: at 599, 719 and $1253 \mathrm{~cm}^{-1}$. The latter one, the most intense, is also the peak with the highest wavenumber among the ones here considered and it correspond to $\mathrm{N}_{i}$-Si bond stretching. The $599 \mathrm{~cm}^{-1}$ mode is connected only with $\mathrm{N}_{s}$, while in the $719 \mathrm{~cm}^{-1}$ mode both $\mathrm{N}$ atoms are involved. 
The bottom panels refer to the most complex defect, and presents four defect intense IR peaks: at 785, 817, 925 and $1002 \mathrm{~cm}^{-1}$, and involve the motion of the three defect atoms. The Raman spectra of all but top case of the figure are dominated by the perfect bulk peak. Defect peaks have quite low intensity, at least one order of magnitude lower than the one of perfect silicon.

\section{The $I_{N-N}, I_{N-S i}, I_{C-N}$ and $I_{S i-S i}$ defects}

The left top panel of figure 9 shows the IR spectrum of the $\mathrm{I}_{\mathrm{N}-\mathrm{N}}$ defect. It is dominated by two peaks at 584 and $778 \mathrm{~cm}^{-1}$. The former, of $\mathrm{B}_{2}$ symmetry, is the antisymmetric stretching of the N-N couple along the $\mathrm{C}_{2}$ axis. The latter, a couple of degenerate modes of E symmetry, are bending modes orthogonal to the $\mathrm{C}_{2}$ axis. The intensity of the $\mathrm{E}$ mode is three times larger than the one of $\mathrm{B}_{2}$ symmetry, that in turn is much larger than the one of all other peaks. The Raman spectrum (top right panel of the figure) is dominated by the pristine silicon peak at $533-535 \mathrm{~cm}^{-1}$; the two defect peaks at 585 and $778 \mathrm{~cm}^{-1}$ are also Raman active; a further peak appears at at $976 \mathrm{~cm}^{-1}$ (symmetric stretching of $\mathrm{A}_{1}$ symmetry, only Raman active); these peaks have however intensity that are more than 20 times smaller that the dominant Raman peak. The two central panels of figure 9 refer to $\mathrm{I}_{\mathrm{N}-\mathrm{Si}}$. The peak that in $\mathrm{I}_{\mathrm{N}-\mathrm{N}}$ is at 585 red shifts by $30 \mathrm{~cm}^{-1}$ to $555 \mathrm{~cm}^{-1}$. The one at $778 \mathrm{~cm}^{-1}$ in $\mathrm{I}_{\mathrm{N}-\mathrm{N}}$ blue shifts by $12 \mathrm{~cm}^{-1}$ to $790 \mathrm{~cm}^{-1}$. Due to the lower symmetry, the in phase N-Si stretching is now IR active, and appears at $887 \mathrm{~cm}^{-1}$ (as a consequence of the larger mass of $\mathrm{Si}$ ). For symmetry reasons, all these peaks are both IR and Raman active. The Raman spectrum (panel to the right) is also in this case dominated by the perturbed pristine silicon peak at $531-535 \mathrm{~cm}^{-1}$.

We also considered the $\mathrm{I}_{\mathrm{C}-\mathrm{N}}$ defect, containing two heteroatoms. The IR and Raman spectra are shown in the bottom panels in figure 9. At variance with respect to $\mathrm{I}_{\mathrm{N}-\mathrm{Si}}$, the system maintains the $\mathrm{C}_{2 v}$ symmetry. The antisymmetric stretching is now at $590 \mathrm{~cm}^{-1}$; the in plane modes, that were degenerate in $\mathrm{I}_{\mathrm{N}-\mathrm{N}}$ at $778 \mathrm{~cm}^{-1}$, red shift to 762 and $766 \mathrm{~cm}^{-1}$; the in phase stretching moves up to $1028 \mathrm{~cm}^{-1}$. As usual, the Raman spectrum shows the defect modes with very low intensity. For completeness, also the $\mathrm{I}_{\mathrm{Si}-\mathrm{Si}}$ case has been considered. Here, the two possible spin states, namely the triplet $t$ and the singlet $s$ have been investigated, whose IR spectra are shown in figure 10. We note that: 
a) The $t$ and $s$ spectra are very similar, with differences equal to $10,2,4$ and $0 \mathrm{~cm}^{-1}$ for the four most intense peaks shown in the figure.

b) As expected, the highest wavenumber, due to the presence of $\mathrm{N}$ or $\mathrm{C}$ in the cases discussed above, lowers here by about $200 \mathrm{~cm}^{-1}$.

c) The modes related to the two interstitial atoms are above the pure bulk silicon mode at $533 \mathrm{~cm}^{-1}$. Three main peaks appear around $533-537 \mathrm{~cm}^{-1}, 558-560 \mathrm{~cm}^{-1}$, and 588-598 $\mathrm{cm}^{-1}$, where the first number refers to the singlet $s$, and the second to the triplet $t$.

d)The intensity of these modes are up to two order of magnitude lower than the ones of the interstitial defects involving $\mathrm{N}$ (or C), so that they are hardly visible, in particular if defects containing $\mathrm{C}$ or $\mathrm{N}$ are present. This low intensity is due to the low polarity of the Si-Si bonds, with respect to the N-Si, N-N, N-C ones. As usual, the Raman spectra are dominated by the pristine silicon peak, at about $530 \mathrm{~cm}^{-1}$. All defect peaks have negligible intensity.

\section{DISCUSSION AND COMPARISON WITH EXPERIMENTS AND PREVI- OUS CALCULATIONS.}

As usual, the association of a given microscopic structure to a particular IR (or Raman, or EPR) peak is not simple, and is frequently a matter of long debate.

In 1983 Stein $^{12}$ published the IR spectra of nitrogen implanted crystalline silicon. The spectra, collected at $300 \mathrm{~K}$, are characterized by two absorption bands at 764 and $962 \mathrm{~cm}^{-1}$. Isotopic effects, obtained by implanting ${ }^{15} \mathrm{~N}$ instead of ${ }^{14} \mathrm{~N}$, produced a red shift of 16 and $25 \mathrm{~cm}^{-1}$, to 748 and $937 \mathrm{~cm}^{-1}$, confirming then the attribution of these peaks to nitrogen defects. Two of the samples used for the IR experiments were also used for the EPR investigation by Brower ${ }^{11}$. The defect responsible of the EPR signal, named SL5, was attributed to substitutional N by Brower. Stein ${ }^{12}$, discussing Brower's attribution, considers the two following hypotheses: the two signals at 764 and $962 \mathrm{~cm}^{-1}$ are due to two different defects, or to a single defect. In Figure 3 of reference 12, Stein's first hypothesis is graphically represented: the two peaks are attributed to (using our notation) $\mathrm{N}_{i}$ (the low wavenumber one) and to $\mathrm{N}_{i}-\mathrm{N}_{i}$.

In 1985 Stein ${ }^{13}$ published a second paper devoted to ${ }^{14} \mathrm{~N}$-implanted Si. This time the discussion involves a larger set of peaks (see figure 2 of the paper): to the two already discussed, 
one peak at $551 \mathrm{~cm}^{-1}$, that does not shift when ${ }^{14} \mathrm{~N}$ is substituted by ${ }^{15} \mathrm{~N}$; a second one at 653 (shifts by $16 \mathrm{~cm}^{-1}$ to 637 ); and a third one at 687 (shifts by $19 \mathrm{~cm}^{-1}$ to 668 ) must be added. In this second paper, on the basis of various evidences, the peak at $653 \mathrm{~cm}^{-1}$ was attributed to $\mathrm{N}_{i}$ and then associated also to the SL5 EPR signal registered by Brower ${ }^{11}$. Nine years later (1994) Jones et $a .^{14}$, in a mixed experimental-theoretical investigation, observed the same high wavenumber peaks of Stein (at 962 and $765 \mathrm{~cm}^{-1}$ ) that they attributed to the "nitrogen pair defect" (see Table I and figure 3 of ref. 14; in our notation the $\mathrm{N}_{i}-\mathrm{N}_{i}$ defect). Their simulations reported the $\mathrm{B}_{u}$ symmetry modes at 918 and $688 \mathrm{~cm}^{-1}$, with differences of 43 and $76 \mathrm{~cm}^{-1}$ with respect to experiment. Their computed defect wavenumber for nitrogen substitutional differs by $24 \mathrm{~cm}^{-1}$ from the experiment ${ }^{13}$ (677 vs $653 \mathrm{~cm}^{-1}$ ), suggesting a relatively low accuracy of the adopted computational tools.

In 2003 Goss et al. ${ }^{15}$, investigated ten different nitrogen defects in silicon, with a periodic supercell approach, a GGA type functional, a Gaussian type basis set (plus peudopotentials for core electrons) and a non public code. The vibrational modes of most of these defects have been computed, and are reported in Table II, to the right, for comparison with our results (to the left). The ${ }^{14} \mathrm{~N}$ to ${ }^{15} \mathrm{~N}$ isotopic shifts obtained by Goss and in the present paper are also reported. In the following discussion the IR intensities, that have not been computed by Goss et al. ${ }^{15}$, but are listed in Table II and shown in Figures 8 and 9, play obviously a crucial role (is the peak visible? what is the relative intensity of the various peaks? remember that symmetry allowed transitions can have negligible intensities).

We are now in the position to discuss the attribution of the five Stein's peaks. We must however do a preliminary remark. It is clear that, also assuming that experiments are not affected by errors, simulation cannot reproduce exactly the experimental spectra, as the many ingredients of the model (adopted DFT functional, quality of the basis set, numerical accuracy of the adopted computer code) introduce an error that is different for each computed property, is hardly evaluated and is not usually reported in the theoretical papers.

As regards the present study, we observe that the experimental Raman peak of pristine silicon at $521 \mathrm{~cm}^{-1}$ is computed at $533 \mathrm{~cm}^{-1}$ with the present basis set and functional. This might suggest that all peaks in the present calculations are a) overestimated, and b) by an amount that should be not too far from $12 \mathrm{~cm}^{-1}$.

We start the comparison from the two peaks at high wavenumbers, namely at 765 and 962 $\mathrm{cm}^{-1}$. 
As regards the latter, the $\mathrm{N}_{i}-\mathrm{N}_{i}$ peak at 971 is not only just $9 \mathrm{~cm}^{-1}$ above the experiment, but is also extremely intense $(3884 \mathrm{~km} / \mathrm{mol}$, the most intense of this spectrum). The calculated isotopic shift $\left(26 \mathrm{~cm}^{-1}\right)$ coincide exactly with the one by Stein. Very similar numbers have been obtained by Goss $\left(968 \mathrm{~cm}^{-1}\right.$, isotopic shift of $\left.27 \mathrm{~cm}^{-1}\right)$. A careful inspection of Table II shows that this attribution is unique, as there are no additional peaks of other defects in that region.

Let us consider now Stein's peak at $765 \mathrm{~cm}^{-1}$; the second most intense calculated peak of $\mathrm{N}_{i}-\mathrm{N}_{i}$ is at $782 \mathrm{~cm}^{-1}, 17 \mathrm{~cm}^{-1}$ above the experiment. The isotopic shift is $18 \mathrm{~cm}^{-1}\left(16 \mathrm{~cm}^{-1}\right.$ in Stein's paper). The intensity is high $(2723 \mathrm{~km} / \mathrm{mol})$. In Goss paper, this peak is at 773 $\mathrm{cm}^{-1}$, and the shift is equal to $18 \mathrm{~cm}^{-1}$.

One can consider other candidates for these peaks, that may be not produced by a single type of defect, but rather a combination of defects. The $\mathrm{I}_{N-N}$ defect presents an intense peak $(2241 \mathrm{~km} / \mathrm{mol})$ at $778 \mathrm{~cm}^{-1}$, just $13 \mathrm{~cm}^{-1}$ above the experiment, with an isotopic shift of $21 \mathrm{~cm}^{-1}$. It is difficult to rule out this second option.

The lowest wavenumber peak in the Stein list is at $551 \mathrm{~cm}^{-1}$, and does not shift under the ${ }^{15} \mathrm{~N}$ to ${ }^{14} \mathrm{~N}$ substitution. The only simulated peak in this region is the one of $\mathrm{N}_{i}-\mathrm{N}_{i}$ at 563 $\mathrm{cm}^{-1}\left(12 \mathrm{~cm}^{-1}\right.$ above the experiment), that shifts by just one $\mathrm{cm}^{-1}$ under isotopic substitution. Its intensity is $800 \mathrm{~km} / \mathrm{mol}$, about $25 \%$ of the dominant (calculated) peak at 971 $\mathrm{cm}^{-1}$, clearly visible in Figure 8. Other candidates, with wavenumbers between 550 and 590 $\mathrm{cm}^{-1}$, must be ruled out (see Table II) because of the low intensity and/or because of the non null shift under nitrogen isotopic substitution. Note that this mode does not appear in Table II of Reference 15, devoted to the $\mathrm{N}_{i}$ - $\mathrm{N}_{i}$ defect.

In summary, three out of five peaks of Stein's list can be attributed with high probability to the $\mathrm{N}_{i}-\mathrm{N}_{i}$ defect. This attribution is supported by the observation that this defect is the most stable one among the ones here considered.

The two remaining peaks at 653 and $687 \mathrm{~cm}^{-1}$ have already been discussed in a parallel paper ${ }^{17}$ devoted to a set of substitutional defects, isolated or aggregated and combined with vacancies. The attribution to $\mathrm{N}_{s}$ of the former, and to $\mathrm{N}_{s}-\mathrm{N}_{s}$ of the latter is partially confirmed by our simulated values at $645 \mathrm{~cm}^{-1}, 8 \mathrm{~cm}^{-1}$ below the experimental peak, and at $685 \mathrm{~cm}^{-1}$, just $2 \mathrm{~cm}^{-1}$ below the experiment. We can repeat also in this case the question: are there other candidates? The $\mathrm{VN}_{1}^{d}$ defect (a substitutional nitrogen sitting on the border of a vacancy, in its lowest energy doublet state) presents a peak at $664 \mathrm{~cm}^{-1}\left(11 \mathrm{~cm}^{-1}\right.$ above 
the experiment), with high intensity $(1782 \mathrm{~km} / \mathrm{mol})$. There is also a second peak at 673 $\mathrm{cm}^{-1}$, with high intensity $(1689 \mathrm{~km} / \mathrm{mol})$ that might merge with the previous one to give a single band at about $16 \mathrm{~cm}^{-1}$ above the experiment.

Also for Stein's peak at $687 \mathrm{~cm}^{-1}$ there are other candidates, involving $\mathrm{N}$ atoms and a vacancy: $\mathrm{VN}_{2}$ in its singlet ground state presents an intense $(3299 \mathrm{~km} / \mathrm{mol})$ peak at 693 $\mathrm{cm}^{-1}\left(6 \mathrm{~cm}^{-1}\right.$ above the experiment). In $\mathrm{VN}_{3}^{d}$ there are three peaks at $687,694,696 \mathrm{~cm}^{-1}$, that probably would appear as a single peak, and whose total intensity is as large as 11000 $\mathrm{km} / \mathrm{mol}$. These various candidates, that present peaks in a very narrow window, are expected to contribute to the spectrum, with percentages that are difficult to define.

\section{CONCLUSIONS.}

Seven different point defects containing nitrogen atoms in interstitial positions have been investigated by using a supercell model, a local basis set, the B3LYP functional and the CRYSTAL code. The defect geometry has been optimized, the electronic structure analyzed, and the IR and Raman spectra generated. The main aim was to characterize the various defects, and to attribute the five peaks appearing in the IR spectra produced by Stein ${ }^{12,13}$ in samples in which the $\mathrm{N}$ content has been augmented by implantation techniques, so as to generate a reasonably intense IR signal.

Crucial to this analysis is the availability of a code able to generate both the wavenumbers and the IR (as well as Raman) intensities. At variance with respect to previous studies ${ }^{14,15}$, these intensities are computed (analytically), so that it has been possible to generate the full IR and Raman spectra. Other complementary tools, such as the isotopic substitution and the graphical animation of the modes, permit a complete characterization of the peaks of interest.

To all the five peaks reported by Stein it has been possible to associate at least one simulated counterpart. More explicitly:

a) the experimental peak at $551 \mathrm{~cm}^{-1}$ can be attributed to $\mathrm{N}_{i}-\mathrm{N}_{i}$, with a simulated peak at $563 \mathrm{~cm}^{-1}$, and intensity of $800 \mathrm{~km} / \mathrm{mol}$. Both the experimental and calculated isotopic shifts (IS) are null.

b) for the experimental peak at $653 \mathrm{~cm}^{-1}\left(\mathrm{IS}=16 \mathrm{~cm}^{-1}\right)$ we propose two candidates: the $\mathrm{N}_{s}$ peak at $645 \mathrm{~cm}^{-1}\left(3425 \mathrm{~km} / \mathrm{mol}\right.$; IS $\left.=19 \mathrm{~cm}^{-1}\right)$ and the $\mathrm{VN}_{1}^{d}$ peak at $664(1782 \mathrm{~km} / \mathrm{mol}$; 
IS $\left.=18 \mathrm{~cm}^{-1}\right)$.

c) for the experimental peak at $687 \mathrm{~cm}^{-1}\left(\mathrm{IS}=19 \mathrm{~cm}^{-1}\right)$ there are three candidates: i) the $\mathrm{N}_{s}-\mathrm{N}_{s}$ peak at $685 \mathrm{~cm}^{-1}\left(6854 \mathrm{~km} / \mathrm{mol}\right.$; IS $\left.\left.=18 \mathrm{~cm}^{-1}\right)\right)$; ii) the $\mathrm{VN}_{2}$ peak at $693 \mathrm{~cm}^{-1}(3299$ $\mathrm{km} / \mathrm{mol}$; IS $=17 \mathrm{~cm}^{-1}$ ) and the $\mathrm{VN}_{3}$ peak at about $694 \mathrm{~cm}^{-1}$ (three peaks in $9 \mathrm{~cm}^{-1}$ with a total intensity of $11000 \mathrm{~km} / \mathrm{mol}$ and a mean IS value of $\left.18 \mathrm{~cm}^{-1}\right)$.

d) for the experimental peak at $766 \mathrm{~cm}^{-1}\left(\mathrm{IS}=18 \mathrm{~cm}^{-1}\right)$ there are two possible contributions: the $782 \mathrm{~cm}^{-1}$ peak of $\mathrm{N}_{i^{-}} \mathrm{N}_{i}\left(2723 \mathrm{~km} / \mathrm{mol}\right.$; IS $\left.=18 \mathrm{~cm}^{-1}\right)$, and the $778 \mathrm{~cm}^{-1}$ peak of $\mathrm{I}_{N N}$ $\left(2241 \mathrm{~km} / \mathrm{mol} ; \mathrm{IS}=19 \mathrm{~cm}^{-1}\right)$

e) the experimental peak at $963 \mathrm{~cm}^{-1}\left(\mathrm{IS}=26 \mathrm{~cm}^{-1}\right)$ has a calculated counterpart at 971 $\mathrm{cm}^{-1}\left(3484 \mathrm{~km} / \mathrm{mol} ; \mathrm{IS}=26 \mathrm{~cm}^{-1}\right)$ from the $\mathrm{N}_{i}-\mathrm{N}_{i}$ defect.

In summary, quantum mechanical simulation has been shown to be able to identify peaks corresponding to the ones appearing in the experimental spectrum.

In the near future, the extension of the present analysis to the EPR spectra will permit a cross identification of the microscopic nature of many of the $\mathrm{N}$ defects.

\section{ACKNOWLEDGEMENTS}

FSG acknowledge the CINECA award (HP10BZSAHZ and HP10CTG8YY) under the ISCRA initiative, for the availability of high performance computing resources and support. AP and EK acknowledge the framework of the EUROfusion Consortium and have received funding from the Euratom research and training programme 2014-2018 under grant agreement No 633053. The views and opinions expressed herein do not necessarily reflect those of the European Commission. 
Figures 


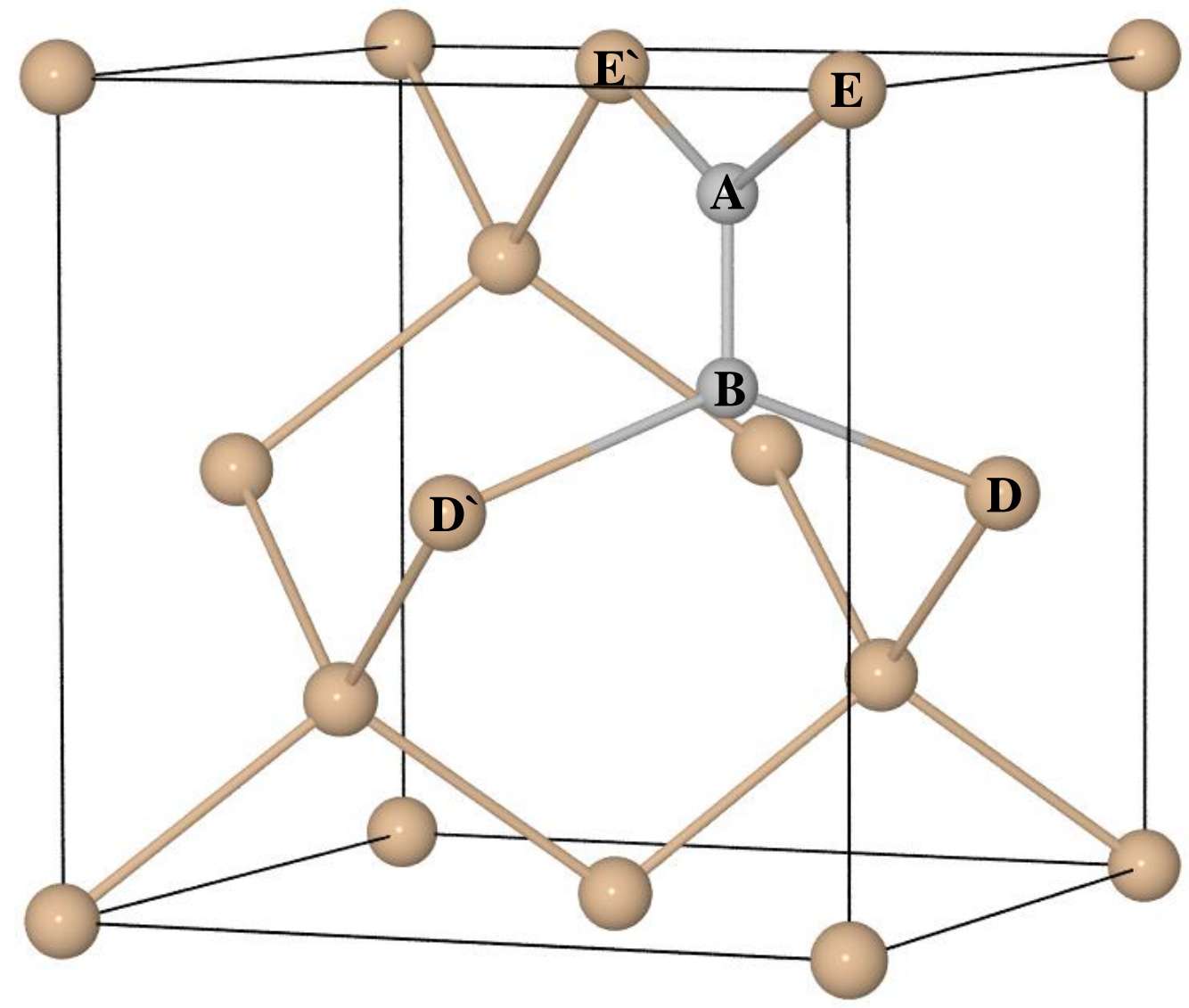

FIG. 1: Schematic representation of the $\langle 001\rangle$ split interstitial defect. The three coordinated A and $\mathrm{B}$ atoms can be silicon or nitrogen. E, E', D and D' are the silicon atoms linked to A and B, respectively. When symmetry reduces to $\mathrm{C}_{2}$ or $\mathrm{C}_{S}$, atoms $\mathrm{E}$ and $\mathrm{E}^{6}$, and $\mathrm{D}$ and $\mathrm{D}^{6}$ are no more equivalent. 


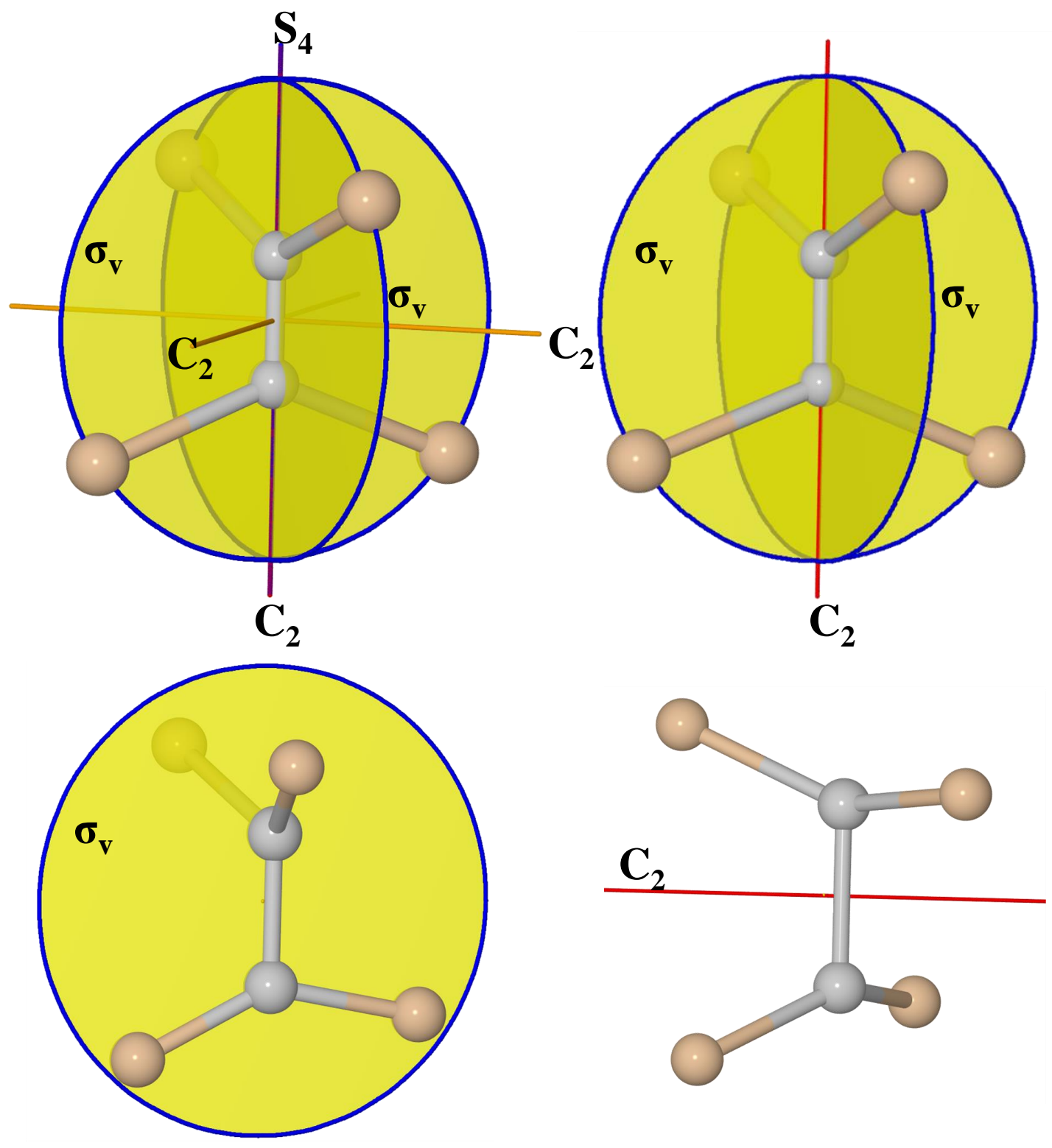

FIG. 2: Point symmetry operators for the $\langle 001\rangle$ split interstitial with $\mathrm{D}_{2 d}, \mathrm{C}_{2 v}, \mathrm{C}_{s}$ and $\mathrm{C}_{2}$ symmetry. 

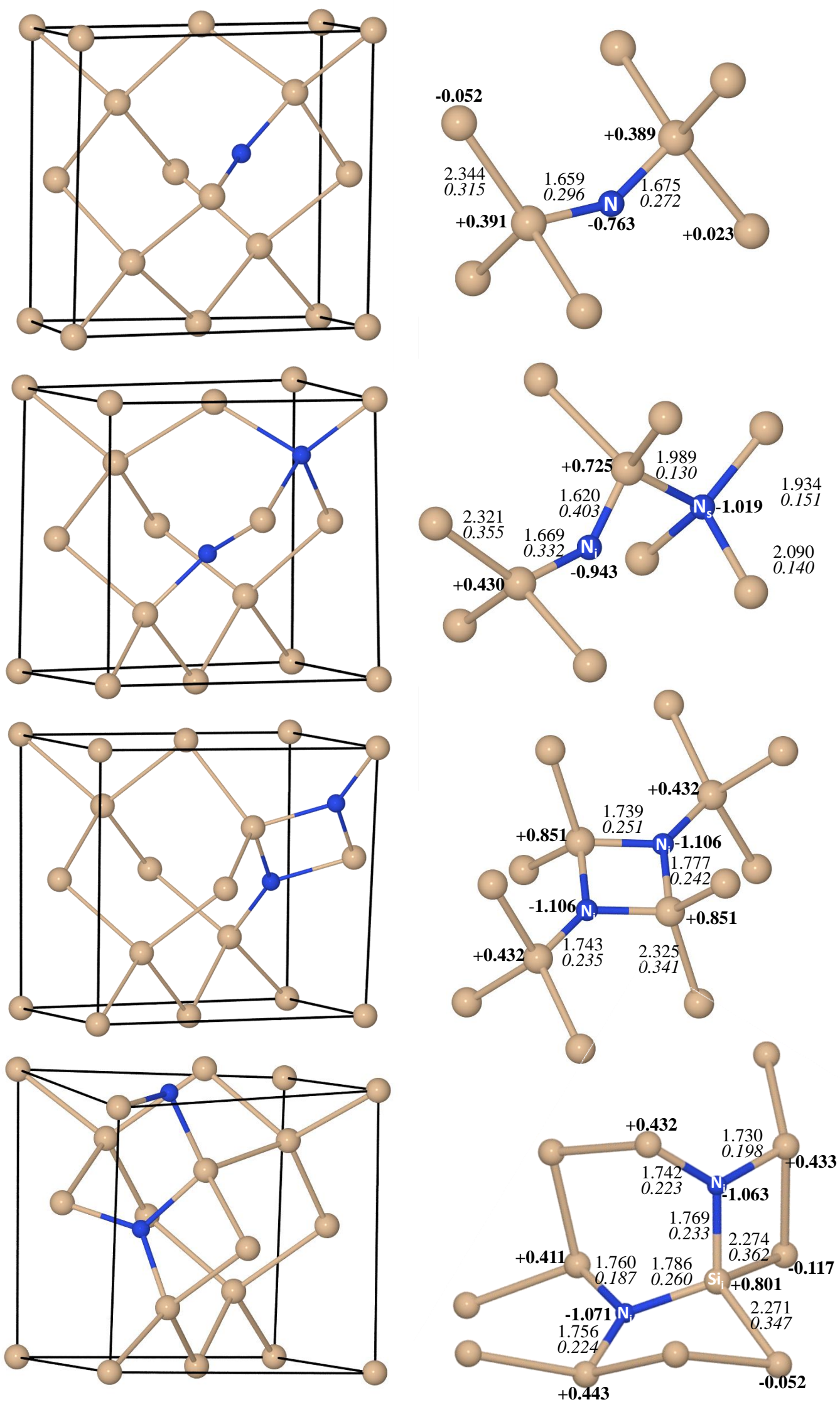
FIG. 3: From top to bottom: $\mathrm{N}_{i}, \mathrm{~N}_{i}-\mathrm{N}_{s}, \mathrm{~N}_{i}-\mathrm{N}_{i}$ and $\mathrm{N}_{i}-\mathrm{Si}_{i}-\mathrm{N}_{i}$ defects in silicon. To the left, the conventional cell; to the right a local cluster around the defect, with Mulliken net charges (in $|e|$ and bold), bond lengths (in $\AA$ ) and bond populations (in $|e|$ and italic). Only data for non equivalent atoms are reported. 


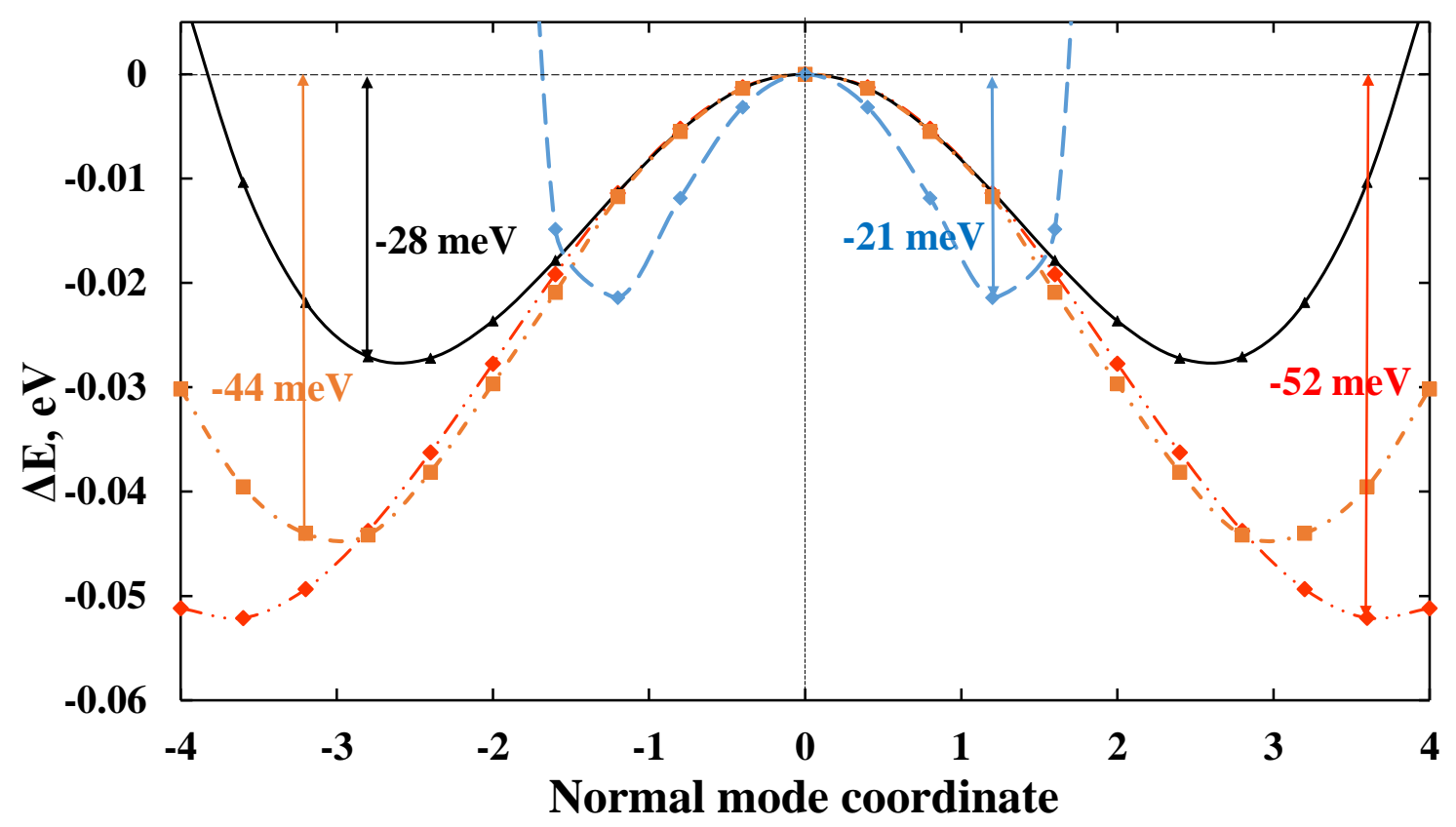

FIG. 4: Energy surface along the imaginary normal mode obtained when imposing the $\mathrm{D}_{2 d}, \mathrm{C}_{2 v}$ or $\mathrm{C}_{3 v}$ symmetry: $\mathrm{I}_{\mathrm{N}-\mathrm{Si}}$ (solid black line), $\mathrm{I}_{\mathrm{Si}-\mathrm{Si}}$ triplet (orange dash-dotted line), and singlet (red dash-dotted line) and $\mathrm{N}_{i}$ (blue line). The imaginary mode has a wavenumber between -140 and $-150 \mathrm{~cm}^{-1}$ for the three $\mathrm{I}_{\mathrm{X}-\mathrm{Y}}$ cases, and close to $-500 \mathrm{~cm}^{-1}$ in the $\mathrm{N}_{i}$ case. 

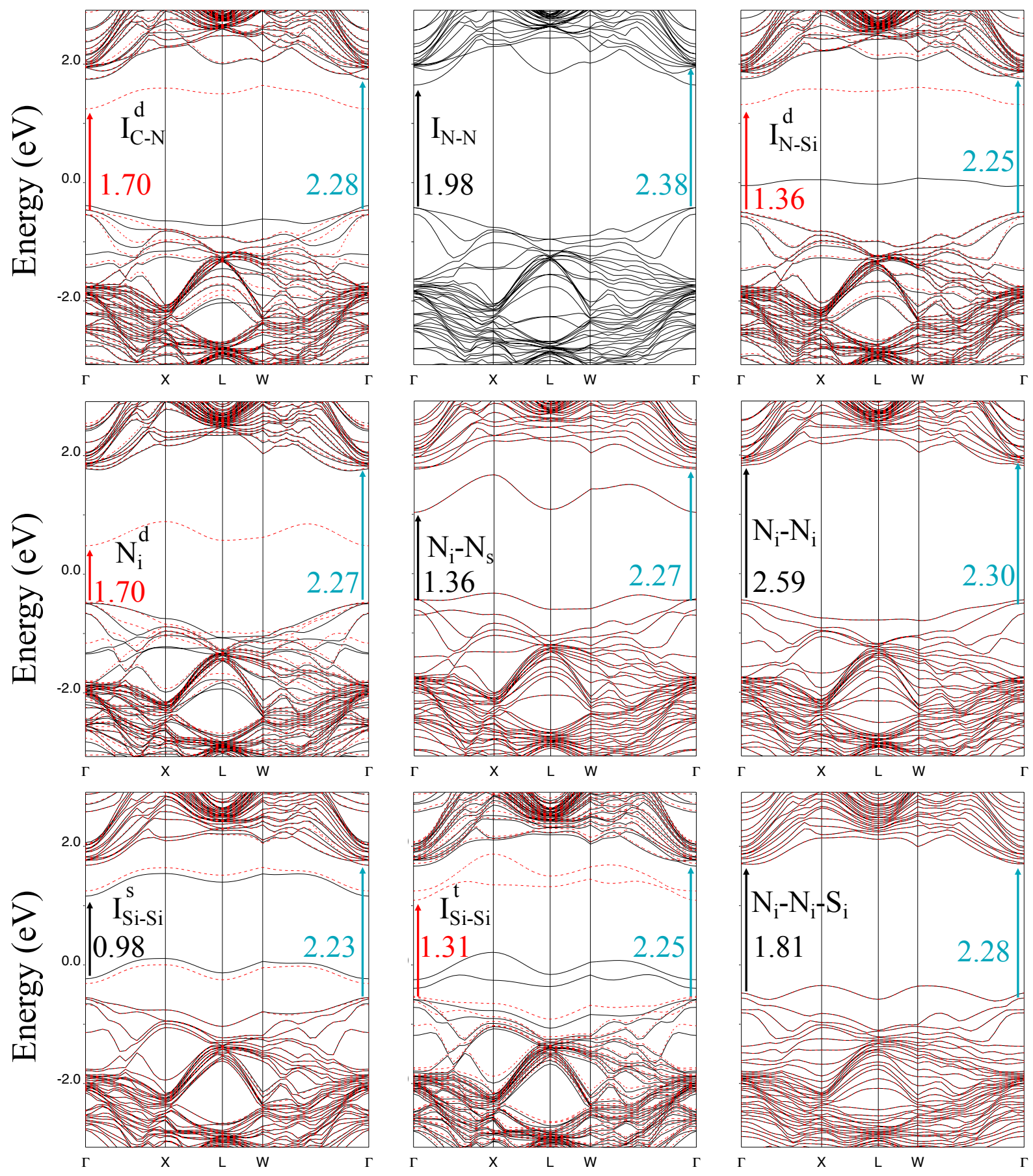

FIG. 5: Band structure of the investigated $\mathrm{N}$ containing defects. The majority spin bands $(\alpha)$ are continuous, the minority spin bands $(\beta)$ are dashed. The pristine silicon band gaps, with the present basis set and functional, are 2.38 (vertical, in $\Gamma$ ) and $1.99 \mathrm{eV}$ (indirect). In all cases the $\mathrm{S}_{64}$ supercell has been used. 


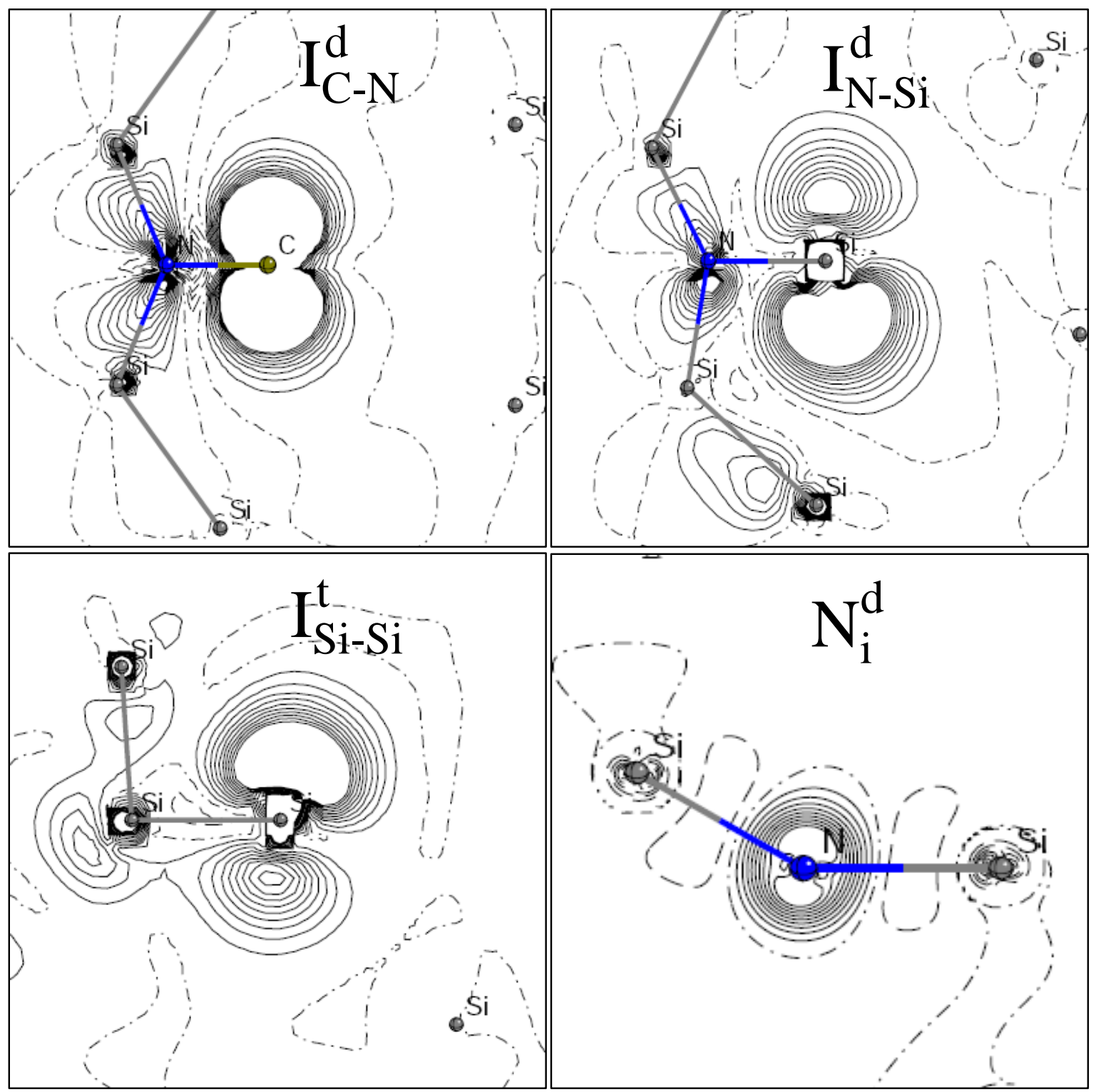

FIG. 6: Spin density maps of various defects in silicon. For the $\mathrm{I}_{\mathrm{X}-\mathrm{Y}}$ defects, atoms $\mathrm{A}, \mathrm{B}$, and D (see Figure 1) define the plane of the figure. For $\mathrm{N}_{i}$, the plane is defined by the nitrogen and its two nearest silicon atoms. Isodensity lines differ by $0.01|e| /\left(a_{0}^{3}\right)$; spin density is truncated at \pm $0.1|e| /\left(a_{0}^{3}\right)$. Continuous, dashed and dot-dashed black lines indicate positive, negative and zero values, respectively. 


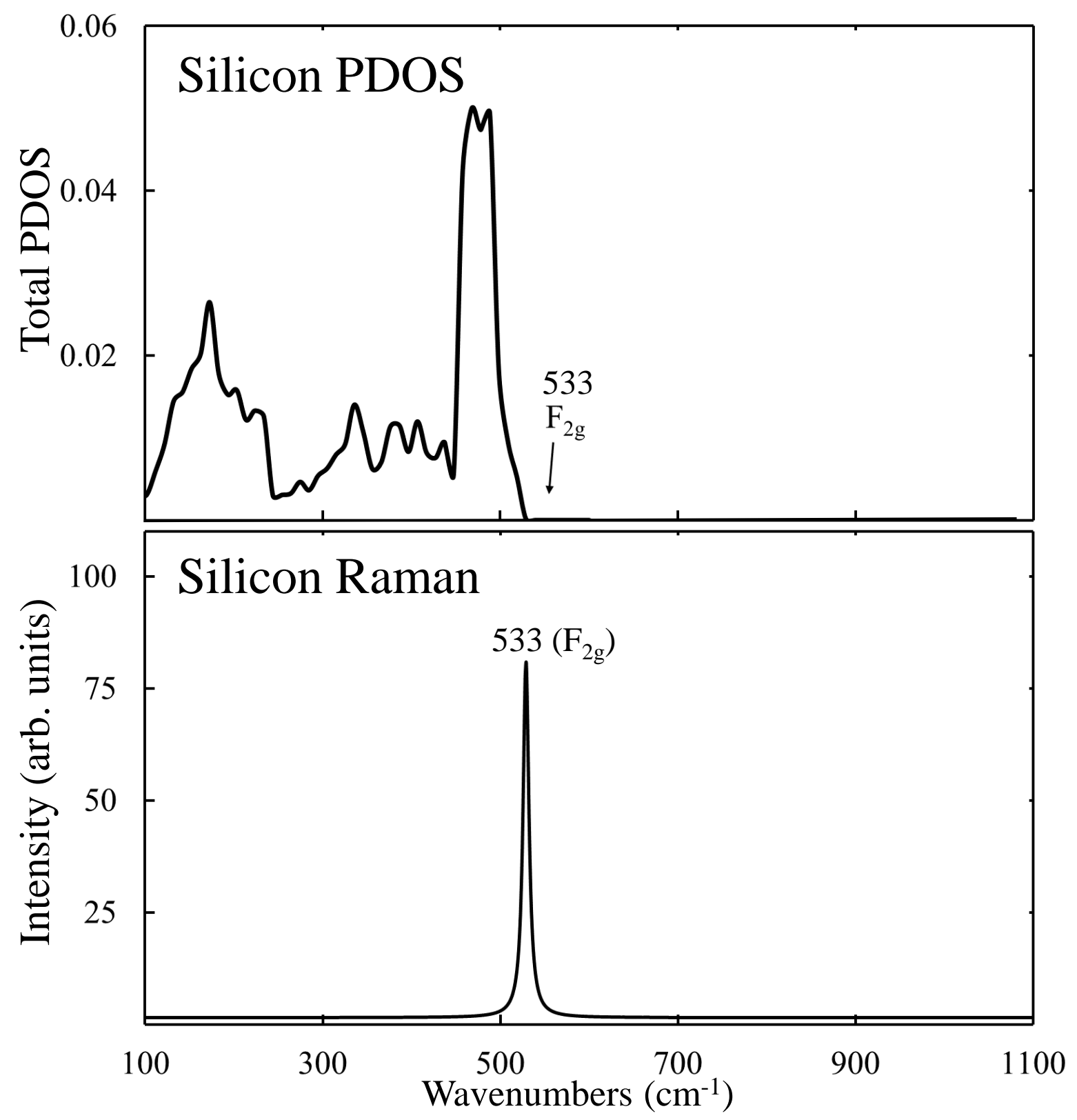

FIG. 7: Total phonon density of state PDOS (top) and Raman spectrum of bulk silicon. The PDOS extends from 24 to $533 \mathrm{~cm}^{-1}$. The Raman peak coincides with the highest eigenvalue. 

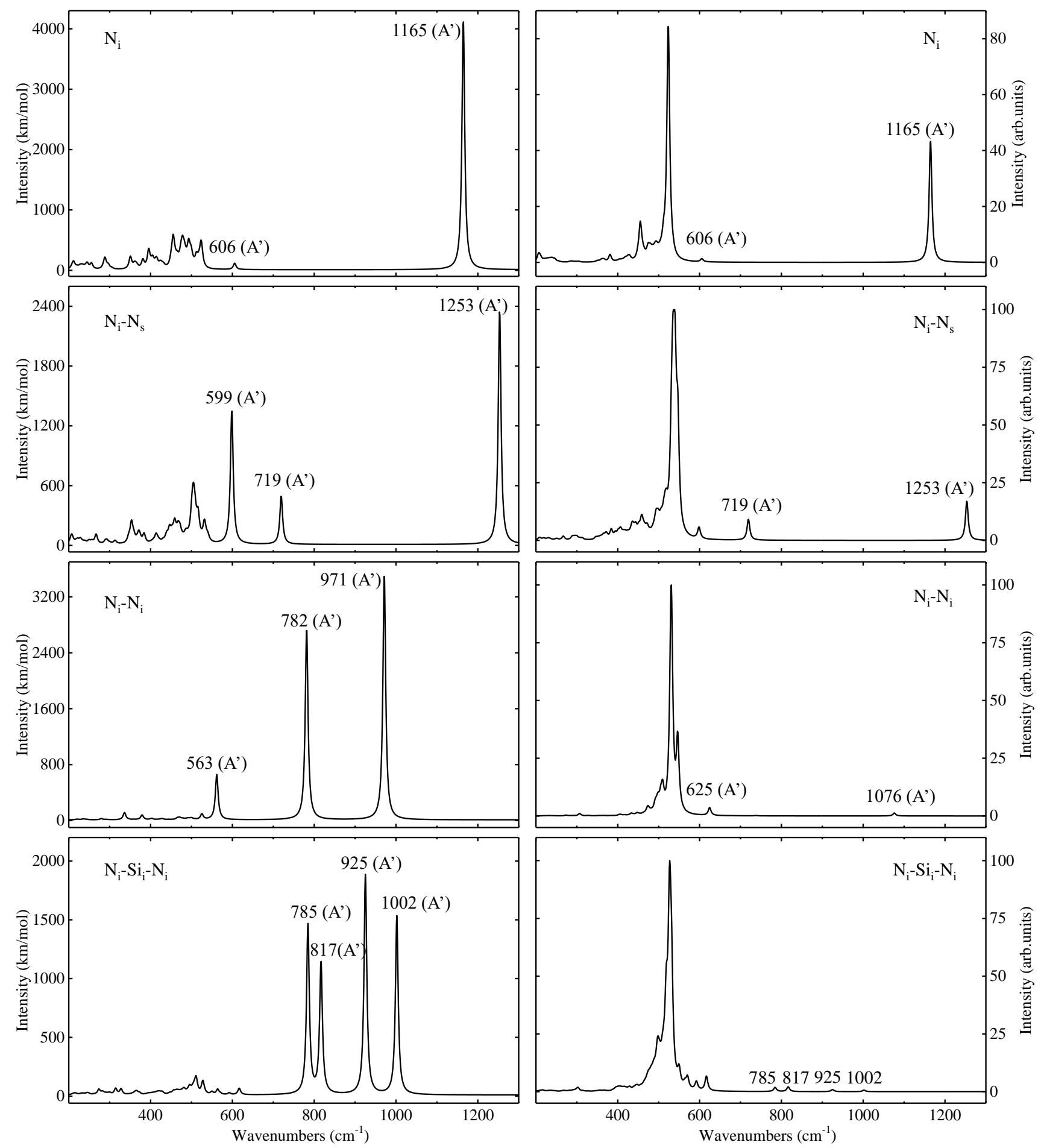

FIG. 8: Simulated IR (left) and Raman (right) spectra of the $\mathrm{N}_{i}, \mathrm{~N}_{s}-\mathrm{N}_{i}, \mathrm{~N}_{i}-\mathrm{N}_{i}$ and $\mathrm{N}_{i}-\mathrm{Si}_{i}-\mathrm{N}_{i}$ defects in silicon. In all cases the $\mathrm{S}_{64}$ supercell has been used. 

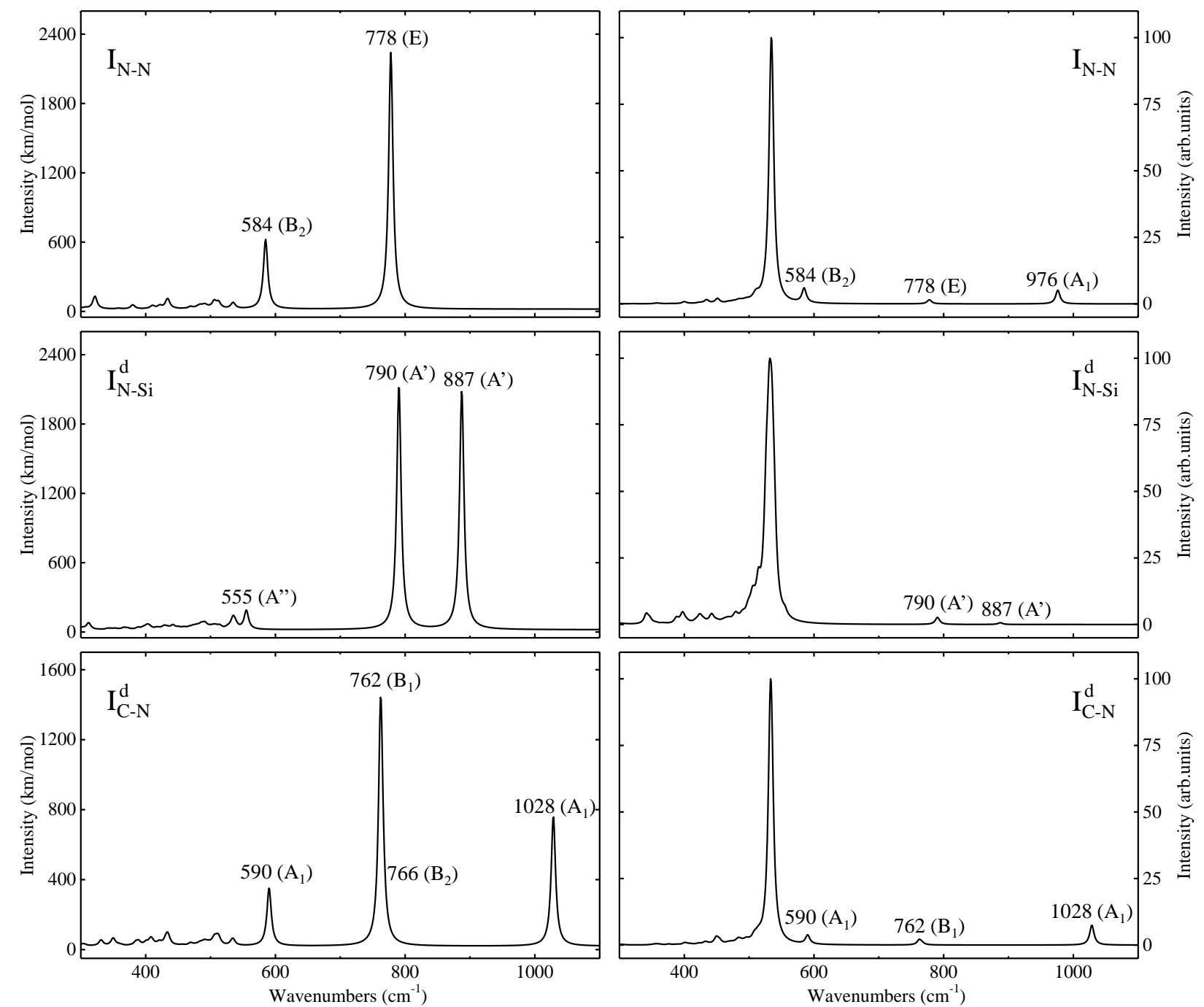

FIG. 9: IR (left) and Raman (right) spectra of $\langle 100\rangle$ split interstitial defects. Superscripts d stand for doublet. All data refer to the $\mathrm{S}_{64}$ supercell. 


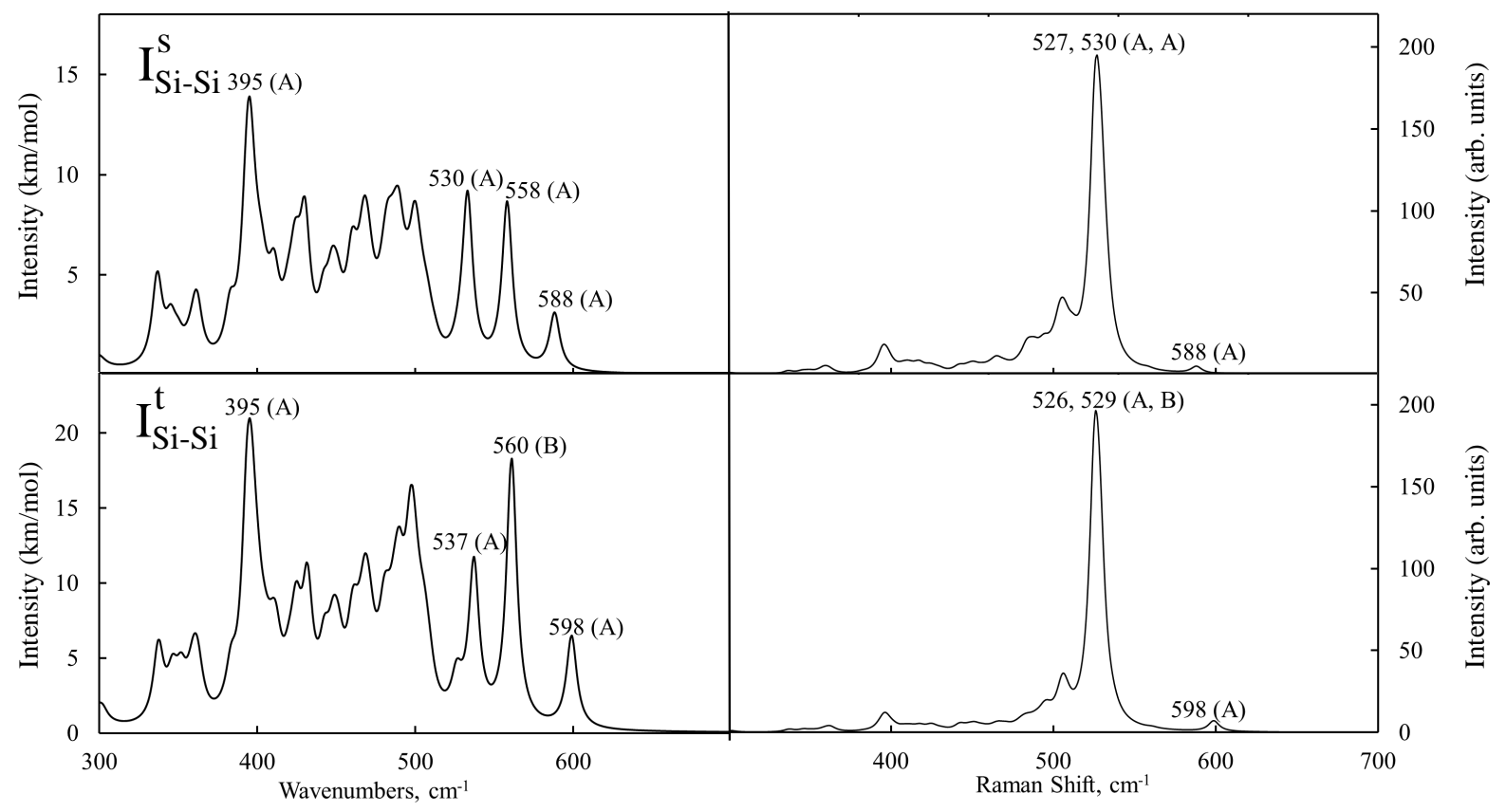

FIG. 10: Simulated IR (left) and Raman (right) spectra of $\mathrm{I}_{\mathrm{Si}-\mathrm{Si}}$ in the singlet $s$ (top) and triplet $t$ (bottom) states. 
Tables 


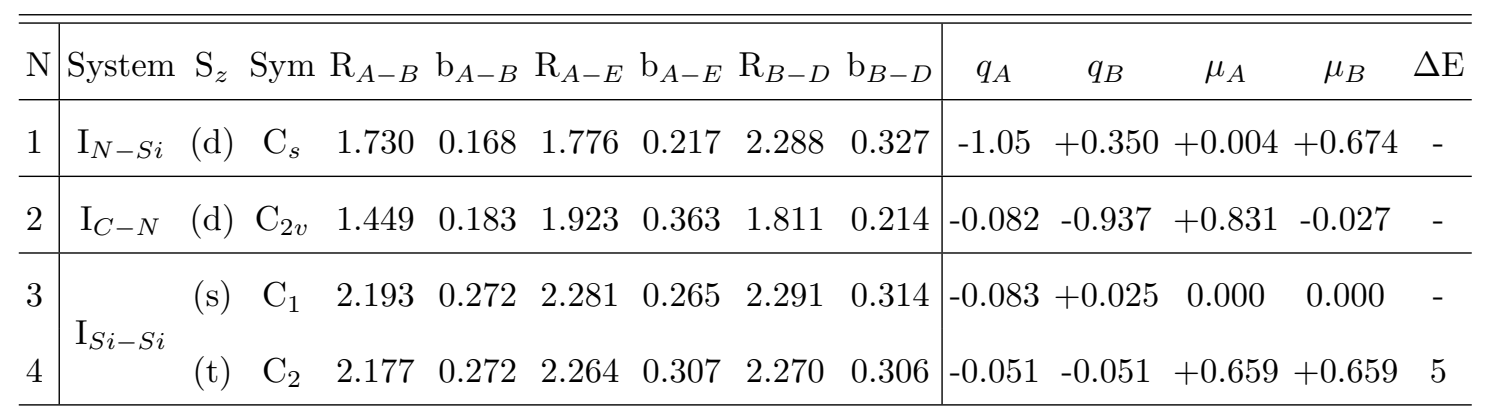

TABLE I: Distances R (in $\AA$ ) between the A and B interstitial atoms and their first neighbors (see figure 1 for the atomic labels), atomic net charges $q$ and magnetic moments $\mu$ of the $\mathrm{A}$ and $\mathrm{B}$ atoms, and bond populations $b$ as resulting from a Mulliken analysis. $q, \mu$ and $b$ in $|e|$. Sym gives the local point symmetry. $s, d, t$ stand for singlet, doublet and triplet $\left(\mathrm{S}_{z}=0,1 / 2\right.$ and 1$) . \Delta \mathrm{E}$ is the energy difference (in meV) between the lowest and highest spin state. All values refer to the $S_{64}$ supercell. 


\begin{tabular}{|c|c|c|c|}
\hline \multirow[t]{2}{*}{ Defect } & & \multicolumn{2}{|l|}{ wavenumber, $\mathrm{cm}^{-1}$ (Sym, Intens) } \\
\hline & & This work & Goss 2003 \\
\hline \multirow{2}{*}{$\mathrm{N}_{i}$} & $\mathrm{~N}^{14}$ & 1165 (4105), $605(103)$ & - \\
\hline & $\mathrm{N}^{15}$ & 1132,604 & \\
\hline \multirow{2}{*}{$\mathrm{N}_{i}-\mathrm{N}_{s}$} & $\mathrm{~N}^{14} \mathrm{~N}^{14}$ & $1253(2332), 719(481), 599(1335)$ & $1163,673,593$ \\
\hline & $\mathrm{N}^{15} \mathrm{~N}^{15}$ & $1219,712,584$ & $1132,665,580$ \\
\hline \multirow{2}{*}{$\mathrm{N}_{i}-\mathrm{N}_{i}$} & $\mathrm{~N}^{14} \mathrm{~N}^{14}$ & 1076 (R), 971 (3484), 782 (2723), 738 (R), 563 (627) & $1070,968,773,743$ \\
\hline & $\mathrm{N}^{15} \mathrm{~N}^{15}$ & $1044,945,764,720,562$ & $1038,941,755,725$ \\
\hline \multirow{2}{*}{$\mathrm{N}_{i}-\mathrm{Si}_{i}-\mathrm{N}_{i}$} & $\mathrm{~N}^{14} \mathrm{~N}^{14}$ & 1002 (1523), 925 (1878), 817 (1120), 784 (1451), 617 (54), 592, 570, 564 & $948,936,835,810,575,570,566$ \\
\hline & $\mathrm{N}^{15} \mathrm{~N}^{15}$ & $947,900,786,765,616,592(15), 570(7), 564(43)$ & $922,911,813,791,575,570,566$ \\
\hline \multirow{2}{*}{$\mathrm{I}_{N-N}$} & $\mathrm{~N}^{14} \mathrm{~N}^{14}$ & $976\left(\mathrm{~A}_{1}, \mathrm{R}\right), 778(\mathrm{E}, 2241), 584\left(\mathrm{~B}_{2}, 623\right)$ & $1003\left(\mathrm{~A}_{1}\right), 774(\mathrm{E}), 573\left(\mathrm{~B}_{2}\right)$ \\
\hline & $\mathrm{N}^{15} \mathrm{~N}^{15}$ & $943\left(\mathrm{~A}_{1}\right), 757(\mathrm{E}), 576\left(\mathrm{~B}_{2}\right)$ & $970\left(\mathrm{~A}_{1}\right), 753(\mathrm{E}), 565\left(\mathrm{~B}_{2}\right)$ \\
\hline \multirow{2}{*}{$\mathrm{I}_{N-S i}$} & $\mathrm{~N}^{14} \mathrm{Si}^{28}$ & $887\left(\mathrm{~A}^{\prime}, 2080\right), 790\left(\mathrm{~A}^{\prime}, 2106\right), 555\left(\mathrm{~A}^{\prime \prime}, 162\right)$ & $885\left(\mathrm{~A}^{\prime}\right), 773\left(\mathrm{~A}^{\prime}\right), 550\left(\mathrm{~A}^{\prime \prime}\right)$ \\
\hline & $\mathrm{N}^{15} \mathrm{Si}^{28}$ & $865\left(\mathrm{~A}^{\prime}\right), 770\left(\mathrm{~A}^{\prime}\right), 555\left(\mathrm{~A}^{\prime \prime}\right)$ & $862\left(\mathrm{~A}^{\prime}\right), 754\left(\mathrm{~A}^{\prime}\right), 550\left(\mathrm{~A}^{\prime \prime}\right)$ \\
\hline \multirow{2}{*}{$\mathrm{I}_{C-N}$} & $\mathrm{C}^{12} \mathrm{~N}^{14}$ & $1028\left(\mathrm{~A}^{\prime}, 758\right), 762\left(\mathrm{~A}^{\prime}, 1423\right), 590\left(\mathrm{~A}^{\prime \prime}, 337\right)$ & - \\
\hline & $\mathrm{C}^{14} \mathrm{~N}^{15}$ & $972\left(\mathrm{~A}^{\prime}\right), 742\left(\mathrm{~A}^{\prime}\right), 576\left(\mathrm{~A}^{\prime \prime}\right)$ & - \\
\hline
\end{tabular}

TABLE II: Defect wavenumbers (in decreasing order) of the most intense IR modes, with their symmetry and absolute intensity (in $\mathrm{km} / \mathrm{mol}$ ). For low-symmetry cases, mode symmetry is not reported. To the right, the wavenumbers obtained by Goss et al. ${ }^{15}$ (only the wavenumbers, as the intensities were not computed). $\mathrm{R}$ indicates that the mode is only Raman active. For space reasons, in the $\mathrm{N}_{i}-\mathrm{Si}_{i}-\mathrm{N}_{i}$ case the intensities of the three lowest wavenumber modes have been reported in the second row. 


\begin{tabular}{l|cccccc}
\hline \hline System & Symm & $\nu$ & $\Delta \mathrm{E}_{\text {scan }}$ & $\Delta \mathrm{E}_{\text {tot }}$ & $\mathrm{D}$ \\
\hline $\mathrm{N}_{i}(\mathrm{~d})$ & $\mathrm{C}_{S}$ & -484 & 0.021 & 0.503 & $\mathrm{~N}$ & 0.494 \\
$\mathrm{I}_{N-S i}(\mathrm{~d})$ & $\mathrm{C}_{S}$ & -149 & 0.028 & 0.040 & $\mathrm{~N}$ & 0.008 \\
& & & & & Si 0.289 \\
$\mathrm{I}_{S i-S i}(\mathrm{~s})$ & $\mathrm{C}_{1}$ & -140 & 0.052 & 0.648 & Si 0.336 \\
& & & & & & Si 0.427 \\
$\mathrm{I}_{S i-S i}(\mathrm{t})$ & $\mathrm{C}_{2}$ & -148 & 0.044 & 0.159 & Si 0.433 \\
& & & & & Si 0.433 \\
\hline
\end{tabular}

TABLE III: Geometry relaxation when the $\mathrm{D}_{2 d}$ (Si-Si triplet), $\mathrm{C}_{2 v}$ (N-Si, Si-Si singlet) or $\mathrm{C}_{3 v}$ $\left(\mathrm{N}_{i}\right)$ symmetry constraint is removed. The final point group is given in the symm column. When the dymanical matrix is diagonalized under the symmetry constraint, a single imaginary mode is obtained in these four cases, whove wavenumber $\nu\left(\mathrm{cm}^{-1}\right)$ is given in the second column. $\Delta \mathrm{E}_{\text {scan }}$ (in $\mathrm{eV}$ ) is the energy gain obtained following downhill the normal modes with imaginary frequency (see figure 4). When a geometry optimization of the full coordinate space is then performed, a further energy gain is obtained to give $\Delta \mathrm{E}_{\text {tot }}(\mathrm{eV})$, the total gain. $\mathrm{D}$ (in $\AA$ ) is the modulus of the vector joining the position of the indicated atoms after the constrained geometry optimization and the final fully optimized geometry. 


\begin{tabular}{lccc|cccc|cc}
\hline \hline Method & $\mathrm{E}_{D}$ & $\mathrm{n}$ & $\mathrm{m}$ & $\mathrm{E}_{f}^{1}$ & $\mathrm{E}_{f}^{1} / \mathrm{X}$ & $\delta E$ & $\delta E^{15}$ & $\mathrm{E}_{f}^{2}$ & $\mathrm{E}_{f}^{2} / \mathrm{X}$ \\
& $(\mathrm{Ha})$ & & $(\mathrm{eV})$ & $(\mathrm{eV})$ & $(\mathrm{eV})$ & $(\mathrm{eV})$ & $(\mathrm{eV})$ & $(\mathrm{eV})$ \\
\hline $\mathrm{N}_{i}$ & -18580.6170 & 1 & 0 & 2.07 & 2.07 & 2.45 & 2.23 & -2.65 & -2.65 \\
$\mathrm{~N}_{i}-\mathrm{N}_{s}$ & -18345.8667 & 2 & 1 & 2.51 & 1.26 & 1.64 & 1.59 & -6.92 & -3.46 \\
$\mathrm{~N}_{i-\mathrm{N}_{i}}$ & -18635.45569 & 2 & 0 & -0.77 & -0.39 & 0.00 & 0 & -10.21 & -5.1 \\
$\mathrm{~N}_{i-\mathrm{Si}_{i}-\mathrm{N}_{i}}$ & -18924.80199 & 2 & -1 & 2.54 & 1.27 & 1.66 & 1.25 & -6.89 & -3.45 \\
\hline $\mathrm{I}_{N-N}$ & -18345.90234 & 2 & 1 & 1.54 & 0.77 & 1.16 & 0.99 & -7.89 & -3.94 \\
$\mathrm{I}_{S i-N}^{d}$ & -18580.6239 & 1 & 0 & 1.88 & 1.88 & 2.27 & 1.83 & -2.84 & -2.84 \\
$\mathrm{I}_{C-N}^{d}$ & -18329.19547 & $2 *$ & 1 & 3.21 & 3.21 & 3.60 & - & -1.51 & -1.51 \\
$\mathrm{I}_{S i-S i}^{s}$ & -18815.21106 & 0 & -1 & 5.87 & - & - & - & - & - \\
$\mathrm{I}_{S i-S i}^{t}$ & -18815.21122 & 0 & -1 & 5.87 & - & - & - & - & - \\
\hline \hline
\end{tabular}

TABLE IV: Formation energy $\mathrm{E}_{f}^{1}$ (in $\mathrm{eV}$ ) of the defects in silicon (see equation 5), computed with reference to the $\mathrm{N}_{2}$ molecule and the perfect silicon bulk total energies. $n$ and $m$ are the coefficients that appear in the equation. Note that they can be positive or negative. $\mathrm{E}_{f}^{1} / \mathrm{X}$ same as $\mathrm{E}_{f}^{1}$, but per $\mathrm{N}$ atom. $\delta E$ : same as $\mathrm{E}_{f}^{1} / \mathrm{X}$, but evaluated with respect to the $\mathrm{N}_{i}-\mathrm{N}_{i}$ defect. $\delta E^{15}$ is the same quantity as evaluated by Goss el al. in reference 15. $\mathrm{E}_{f}^{2}$ is the formation energy evaluated with respect to the isolated atoms, and differs from $\mathrm{E}_{f}^{1}$ by $4.72 \mathrm{eV}$ per nitrogen atom (half the $\mathrm{N}_{2}$ binding energy). Data refer to the silicon supercell containing 64 atoms before the defect formation, whose energy is $-18525.95870\left(-289.46810 \mathrm{Ha}\right.$ per atom). The energy of the $\mathrm{N}_{2}$ molecule is $-109.46852 \mathrm{Ha}$ (54.73426 Ha per atom). 
1 Takao Abe and Hiroshi Takeno. Dynamic behavior of intrinsic point defects in $\mathrm{fz}$ and cz silicon crystals. MRS Proceedings, 262:3, 1992.

2 T Sinno, E Dornberger, W von Ammon, R.A Brown, and F Dupret. Defect engineering of czochralski single-crystal silicon. Materials Science and Engineering: R: Reports, 28(5):149198,2000 .

3 V Orlov, H Richter, A Fischer, J Reif, T Müller, and R Wahlich. Mechanical properties of nitrogen-doped cz silicon crystals. Materials Science in Semiconductor Processing, 5(4):403 407, 2002. EMRS, symposium O.

${ }^{4} \mathrm{Q}$ Sun, KH Yao, HC Gatos, and J Lagowski. Effects of nitrogen on oxygen precipitation in silicon. Journal of applied physics, 71(8):3760-3765, 1992.

5 Yoshiko Itoh, Tadashi Nozaki, Tsumoru Masui, and Takao Abe. Calibration curve for infrared spectrophotometry of nitrogen in silicon. Applied Physics Letters, 47(5):488-489, 1985.

${ }^{6}$ Hiroyuki Kageshima, Akihito Taguchi, and Kazumi Wada. Theoretical investigation of nitrogendoping effect on vacancy aggregation processes in si. Applied Physics Letters, 76(25):3718-3720, 2000 .

7 H. Sawada and K. Kawakami. First-principles calculation of the interaction between nitrogen atoms and vacancies in silicon. Phys. Rev. B, 62:1851-1858, Jul 2000.

8 RS Hockett. Anomalous diffusion of nitrogen in nitrogen-implanted silicon. Applied Physics Letters, 54(18):1793-1795, 1989.

9 Xiao Dong, Ning Li, Cong Liang, Haibin Sun, Guojin Feng, Zhen Zhu, Hezhu Shao, Ximing Rong, Li Zhao, and Jun Zhuang. Strong mid-infrared absorption and high crystallinity of microstructured silicon formed by femtosecond laser irradiation in $\mathrm{nf}_{3}$ atmosphere. Applied Physics Express, 6(8):081301, 2013.

10 Xiao Dong, Ning Li, Zhen Zhu, Hezhu Shao, Ximing Rong, Cong Liang, Haibin Sun, Guojin Feng, Li Zhao, and Jun Zhuang. A nitrogen-hyperdoped silicon material formed by femtosecond laser irradiation. Applied Physics Letters, 104(9):091907, 2014.

11 Keith L. Brower. Jahn-teller-distorted nitrogen donor in laser-annealed silicon. Phys. Rev. Lett., 44:1627-1629, Jun 1980.

12 HJ Stein. Vibrational absorption bands for implanted nitrogen in crystalline silicon. Applied 
Physics Letters, 43(3):296-298, 1983.

13 HJ Stein. Infrared absorption band for substitutional nitrogen in silicon. Applied physics letters, 47(12):1339-1341, 1985.

14 R Jones, Sven Öberg, F Berg Rasmussen, and B Bech Nielsen. Identification of the dominant nitrogen defect in silicon. Physical review letters, 72(12):1882, 1994.

15 JP Goss, I Hahn, R Jones, PR Briddon, and Sven Öberg. Vibrational modes and electronic properties of nitrogen defects in silicon. Physical Review B, 67(4):045206, 2003.

16 EA Kotomin and A Popov. Radiation induced defects in simple oxides. Nuclear Instruments and Methods B, 141:1-15, 1998.

17 A Platonenko, F Gentile, F Pascale, M D'Amore, A Ferrari, and R Dovesi. Nitrogen substitutional defects in silicon. a quantum mechanical characterization through the ir modes. Phys. Chem. Chem. Phys., submitted.

18 A. D. Becke. Density-Functional Thermochemistry. III. The Role of Exact Exchange. J. Chem. Phys., 98(7):5648-5652, 1993.

19 C. Lee, W. Yang, and R. Parr. Development of the Colle-Salvetti Correlation-Energy Formula Into a Functional of the Electron Density. Phys. Rev. B, 37(2):785-789, 1988.

20 R. Dovesi, R. Orlando, A. Erba, C. M. Zicovich-Wilson, B. Civalleri, S. Casassa, L. Maschio, M. Ferrabone, M. De La Pierre, P. D'Arco, Y. Noël, M. Causà, M. Rérat, and B. Kirtman. CRYSTAL14: A Program for the Ab Initio Investigation of Crystalline Solids. Int. J. Quantum Chem., 114:1287, 2014.

21 M. M. Francl, W. J. Pietro, W. J. Hehre, J. S. Binkley, M. S. Gordon, D. J. DeFrees, and J. A. Pople. Self-consistent molecular orbital methods. xxiii. a polarization-type basis set for second-row elements. The Journal of Chemical Physics, 77(7):3654-3665, 1982.

22 Ph D'Arco, G Sandrone, R Dovesi, R Orlando, and VR Saunders. A quantum mechanical study of the perovskite structure type of mgsio 3. Physics and Chemistry of Minerals, 20(6):407-414, 1993.

23 M. M. Francl, W. J. Petro, W. J. Hehre, J. S. Binkley, M. S. Gordon, D. J. DeFrees, and J. A. Pople. Self-Consistent Molecular Orbital Methods. XXIII. A Polarization-Type Basis Set for Second-Row Elements. J. Chem. Phys., 77(7):3654-3665, 1982.

24 R. Dovesi, V. R. Saunders, C. Roetti, R. Orlando, C. M. Zicovich-Wilson, F. Pascale, B. Civalleri, K. Doll, N. M. Harrison, I. J. Bush, Ph. D'Arco, and M. Llunell. CRYSTAL 2014 User's 
Manual. . University of Torino, Torino, 2013.

25 F. Pascale, C. M. Zicovich-Wilson, F. Lòpez Gejo, B. Civalleri, R. Orlando, and R. Dovesi. The Calculation of the Vibrational Frequencies of the Crystalline Compounds and its Implementation in the CRYSTAL Code. J. Comput. Chem., 25(6):888-897, 2004.

26 C. M. Zicovich-Wilson, F. Pascale, C. Roetti, V. R. Saunders, R. Orlando, and R. Dovesi. Calculation of the Vibration Frequencies of $\alpha$-Quartz: The Effect of Hamiltonian and Basis Set. J. Comput. Chem., 25(15):1873-1881, 2004.

27 A. Erba, M. Ferrabone, R. Orlando, and R. Dovesi. Accurate Dynamical Structure Factors from Ab Initio Lattice Dynamics: The case of Crystalline Silicon. J. Comput. Chem., 34:346-354, 2013.

28 C. Carteret, M. De La Pierre, M. Dossot, F. Pascale, A. Erba, and R. Dovesi. The Vibrational Spectrum of $\mathrm{CaCO}_{3}$ Aragonite: a Combined Experimental and Quantum-Mechanical Investigation. J. Chem. Phys., 138(1):014201, 2013.

29 J. Baima, M. Ferrabone, R. Orlando, A. Erba, and R. Dovesi. Thermodynamics and Phonon Dispersion of Pyrope and Grossular Silicate Garnets from Ab Initio Simulations. Phys. Chem. Minerals, 43:137-149, 2016.

30 F. Pascale, C. M. Zicovich-Wilson, R. Orlando, C. Roetti, P. Ugliengo, and R. Dovesi. Vibration Frequencies of $\mathrm{Mg}_{3} \mathrm{Al}_{2} \mathrm{Si}_{3} \mathrm{O}_{12}$ Pyrope. An Ab Initio Study with the CRYSTAL Code. J. Phys. Chem. B, 109(13):6146-6152, 2005.

31 G. M. Barrow. Introduction to Molecular Spectroscopy. McGraw-Hill, New York, 1962.

32 B. A. Hess, L. J. Schaad, P. Carsky, and R. Zahradnik. Ab Initio Calculations of Vibrational Spectra and Their Use in the Identification of Unusual Molecules. Chem. Rev., 86:709-730, 1986.

33 L. Maschio, B. Kirtman, R. Orlando, and M. Rérat. Ab Initio Analytical Infrared Intensities for Periodic Systems Through a Coupled Perturbed Hartree-Fock/Kohn-Sham Method. J. Chem. Phys., 137(20):204113, 2012.

34 L. Maschio, B. Kirtman, M. Rérat, R. Orlando, and R. Dovesi. Comment on "Ab Initio Analytical Infrared Intensities for Periodic Systems Through a Coupled Perturbed Hartree-Fock/KohnSham Method" [J. Chem. Phys. 137, 204113 (2012)]. J. Chem. Phys., 139:167101, 2013.

35 L. Maschio, B. Kirtman, M. Rérat, R. Orlando, and R. Dovesi. Ab initio analytical raman intensities for periodic systems through a coupled perturbed hartree-fock/kohn-sham method 
in an atomic orbital basis. i. theory. J. Chem. Phys., 139(16):164101, 2013.

36 L. Maschio, B. Kirtman, M. Rérat, R. Orlando, and R. Dovesi. Ab Initio Analytical Raman Intensities for Periodic Systems Through a Coupled Perturbed Hartree-Fock/Kohn-Sham Method in an Atomic Orbital Basis. II. Validation and Comparison with Experiments. J. Chem. Phys., 139:164102, 2013.

37 M. Ferrero, M. Rérat, R. Orlando, and R. Dovesi. The Calculation of Static Polarizabilities of Periodic Compounds. The Implementation in the CRYSTAL Code for 1D, 2D and 3D Systems. J. Comput. Chem., 29:1450-1459, 2008.

38 M. Ferrero, M. Rérat, R. Orlando, and R. Dovesi. Coupled Perturbed Hartree-Fock for Periodic Systems: The role of Symmetry and Related Computational Aspects. J. Chem. Phys., 128:014110, 2008.

39 J. H. Parker, D. W. Feldman, and M. Ashkin. Raman scattering by silicon and germanium. Phys. Rev., 155:712-714, Mar 1967. 